\title{
Géolinguistique
}

$17 \mid 2017$

Varia

\section{Le traitement dialectométrique du corpus Bourciez de 1895}

Dialectometric Treatment of the Bourciez Corpus from 1895

\section{Gotzon Aurrekoetxea et Charles Videgain}

\section{(2) OpenEdition}

\section{Journals}

\section{Édition électronique}

URL : http://journals.openedition.org/geolinguistique/431

DOI : 10.4000/geolinguistique.431

ISSN : 2650-8176

\section{Éditeur}

UGA Éditions/Université Grenoble Alpes

\section{Édition imprimée}

Date de publication : 1 décembre 2017

Pagination : 49-98

ISBN : 978-2-37747-025-9

ISSN : 0761-9081

\section{Référence électronique}

Gotzon Aurrekoetxea et Charles Videgain, « Le traitement dialectométrique du corpus Bourciez de 1895 », Géolinguistique [En ligne], 17 | 2017, mis en ligne le 01 février 2019, consulté le 31 octobre 2020. URL : http://journals.openedition.org/geolinguistique/431 ; DOI : https://doi.org/10.4000/ geolinguistique.431 


\title{
Le traitement dialectométrique du corpus Bourciez de 1895
}

\author{
Gotzon Aurrekoetxea \\ Université du Pays basque - Gasteiz-Vitoria \\ Charles Videgain \\ Université de Pau et des pays de l'Adour, UMR 5478 CNRS
}

\section{Résumé}

Cette contribution montre la manière d'exploiter à l'aide de la géolinguistique des textes recueillis au XIX ${ }^{\mathrm{e}}$ siècle par É. Bourciez en utilisant différents outils automatiques. Après avoir tapé le manuscrit dans un traitement de texte, nous avons utilisé «Simple Concordance Program» pour créer la liste de mots des 150 textes basques de la collection. Ensuite, nous avons introduit tous les mots dans une base de données et nous avons fait le processus de lemmatisation, avec un programme créé ad hoc, utilisé de manière géolinguistique. Et la dernière étape a consisté à exploiter les données de manière dialectométrique à l'aide du programme VDM conçu par Hans Goebl.

\section{Mots-clés}

Dialectologie, dialectométrie, bases de données, technologie linguistique.

1. Ce programme de travail a bénéficié de l'aide de l'université du Pays basque (VitoriaGasteiz) sous le numéro de projet $00033.130 \mathrm{H}-14888 / 2002$. Cet article a fait l'objet d'une publication dans la revue électronique Dialectologia $\left(\mathrm{n}^{\circ} 2\right.$ 2, 2009, p. 81-111), sous une forme plus réduite; les auteurs ont voulu l'étoffer et le reproposer après autorisation. 


\begin{abstract}
This contribution shows the way to carry out the geolinguistic exploitation of texts gathered in 19th century by É. Bourciez, using different automatic tools. After typing the manuscript in a word processor, we used "Simple Concordance Program" to create the list of words of the 150 Basque texts of the collection. Afterwards, we introduced all of the words in a database and we did the lemmatisation process, with a program made ad hoc, to use in a geolinguistic way. And the final step was to exploit the data in the dialectometric way using the VDM program conceived by Hans Goebl.
\end{abstract}

\title{
Keywords
}

Dialectology, dialectometry, database, linguistic technology.

\section{Introduction}

La dialectologie en domaine basque connaît depuis quelques années divers développements, aussi bien dans le cadre de la dialectologie traditionnelle que depuis une trentaine d'années en ayant recours à des méthodologies plus novatrices. Cela se manifeste en particulier dans l'attention prêtée à la classification des divers parlers ou dialectes. Le travail qui suit présente l'exploitation d'un corpus dialectal datant de la fin du XIX siècle et tente une représentation cartographique des objets linguistiques offerte par un corpus aux items limités en nombre mais d'une grande densité du point de vue géographique. Nous y présentons une cartographie traditionnelle des données (sur support informatisé), puis un traitement inspiré des méthodes de la dialectométrie. Nous avons publié trois ouvrages sur les textes basques recueillis par Édouard Bourciez vers 1895, le premier consacré au corpus lui-même, le second aux cartes lexicales, le troisième aux cartes grammaticales (Aurrekoetxea \& Videgain, 2004; Aurrekoetxea, Videgain \& Iglesias, 2004 [2006], 2005 [2008]). L'aide qui nous a été fournie ${ }^{2}$ nous a permis de réaliser cette trilogie.

2. Nous tenons à remercier particulièrement :

- Paul-Henri Allioux, responsable de la bibliothèque de l'université Bordeaux Montaigne, qui nous a permis de travailler sur le manuscrit Bourciez en nous aidant à résoudre tous les problèmes techniques ou administratifs;

- Bernard Oyharçabal, directeur du laboratoire de recherches du CNRS Iker 5478, sis à Bayonne, et qui a mis les ressources du centre à notre disposition; 


\section{L'édition informatisée des manuscrits}

Au départ (en 2002) l'ensemble des textes recueillis de Bourciez (appelé Recueil Bourciez) a fait l'objet d'un document intitulé Recueil des idiomes de la région Gasconne. Édouard Bourciez réunit en 1894 un total de 4444 versions de la Parabole de l'Enfant prodigue (dont les versions basques). Par le biais des inspecteurs d'académie, il obtint une réponse de tous les instituteurs sollicités, il ne s'agit donc pas d'enseignants simplement volontaires mais de fonctionnaires disciplinés, ce qui explique le quadrillage quasi complet des communes. Grâce à cette collecte, Bourciez réunit un réseau extrêmement serré sur un grand espace et il pensait à juste raison que bien des objets linguistiques seraient saisis dans les mailles de ces nombreuses versions. Par ricochet en quelque sorte, ce souci synchronique fait notre bonheur en domaine basque (même si ne figurent que 150 textes basques sur les 4500 versions au total). Ces manuscrits sont conservés à la bibliothèque de l'université Bordeaux Montaigne.

\subsection{Les manuscrits}

Les 150 versions en langue basque couvrent l'ensemble du Pays basque dans le département des Pyrénées-Atlantiques actuelles (alors département des Basses-Pyrénées). Tous les textes recueillis en Pays basque historique ne sont pas en langue basque : c'est ainsi que les communes suivantes ont fourni des versions occitanes (gascon) à Bayonne (1188-1191), Anglet (1197), Bassussary (1199), Biarritz (1200), Urt (1239), LabetsBiscay (1289), Osserain (1296), Montory (1356). Dans le canton d'Oloron, Esquiule a fourni un texte en basque. Quelques villages n'ont fourni aucun texte, comme à Saint-Martin d'Arrossa, Behorléguy, Gamarthe, IthorrotzOlhaiby ou Berrogain-Laruns. Dans quelques villages, ce sont deux textes qui ont été recueillis, avec des variantes orthographiques ou des différences plus importantes. Au total, ce sont 138 villages qui ont fourni des textes pour le recueil Bourciez. Ce recueil est d'une importance capitale pour la dialectologie basque et la philologie basque. En effet, on ne possède pas beaucoup de séries comparables de textes recueillis presque dans chaque commune. Même s'il s'agit d'une transcription écrite et donc sujette à caution au regard des enquêtes plus récentes faites au magnétophone, nous

- nous ne pouvons que joindre dans ces remerciements la mémoire du professeur Jacques Allières, qui fut notre maître, et l'un des premiers à avoir découvert l'intérêt du recueil Bourciez sur lesquels il publia des articles importants (Allières, 1977, 1986). 
défendons la valeur du témoignage ainsi recueilli : ces instituteurs, sachant ou non le basque, ont généralement fait conserver la qualité de leur traduction par un locuteur connaisseur de la variété de la commune. On sait que la collecte des données a progressé depuis les premières tentatives menées par Georg Wenker en Allemagne qui obtint une célèbre collecte à partir d'une enquête par correspondance. Envoyant son questionnaire par la poste, il ne pouvait à l'époque procéder à l'enquête directe comme on le fait aujourd'hui. La collecte du recueil Bourciez est donc tributaire de son époque et des méthodologies alors en vigueur, cela dit elle demeure de par sa densité et son réseau serré une source de grande valeur. On connaît les dangers et les risques d'une enquête menée en l'absence du linguiste : en particulier, chaque instituteur n'a pas poussé au même degré le souci de refléter les particularités du parler de la commune et certains ont été plus laxistes sur ce point en produisant des formes moins endémiques. Parfois la chose est claire, comme pour la commune de Pagolle où l'instituteur n'a absolument pas suivi le parler et a fourni un texte marqué comme bien plus occidental, peut-être en raison du prestige prêté aux parlers plus éloignés du souletin. Certains ont carrement donné des versions quasi identiques (1268 et 1269). Certes le lecteur attentif relève quelques différences (mithila / muthila, pounitu/punitu, vesta/vestat, edo/ eddo, mais on est certain que les deux textes ont le même auteur, qui utilise certains traits endémiques intéressants comme le futur en hartiko (et non pas *hartuko) ou le verbe ezari (et non pas *ezarri). De toute façon, l'inconvénient est minime puisque les deux textes sont portés comme étant représentatifs l'un et l'autre de Saint-Palais. Le cas de Bunus (1303) et Hosta (1305) est plus piquant, comme nous l'avons montré ailleurs (Videgain, 1989 : 35-38). La solidarité des anciens de l'École normale aidant, il semble que Sagaspe, instituteur de Bunus, ait prêté son texte à l'instituteur Loustau (sans doute béarnais) de Hosta, puisqu'on s'aperçoit que c'est ce dernier qui a copié, non sans faire des erreurs, ce qui laisse entendre qu'il ne comprenait pas le texte qu'il copiait, les fautes s'accumulant en fin de texte, signe d'une attention moins vive : lehin pour lehen, ehaztin pour ehaztun, bezta pour besta. Ici encore le plagiat est sans grande conséquence puisqu'il touche deux communes limitrophes. Le mauvais état de certaines fiches ou leur degré d'illisibilité nous ont poussés à choisir la photographie numérique pure et simple des pages du manuscrit, formatés ensuite en pdf et publiés sous cette forme dans le CD qui accompagne notre ouvrage. On peut aussi les consulter sur Internet sur le site Artxiker du laboratoire Iker ou bien à l'adresse <www.armiarma.com $>$. Chaque texte a été saisi commune par commune, puis l'équipe de travail a corrigé la première version dactylographiée sur Word. La qualité de la copie conditionnait la suite du travail et 
nous avons essayé d'éliminer le plus possible les erreurs de transcription. Un processeur de textes a ensuite permis de réunir toutes les versions dans un document unique. Le résultat en a été le premier volume (Aurrekoetxea $\&$ Videgain, 2004).

\section{Traitement géolinguistique}

\subsection{Du texte à la base de données}

Comme indiqué ci-dessus, les données originales sont organisées commune par commune. Le texte informatisé a été exploité de manière systématique en utilisant des ressources automatisées. L'ensemble du document ayant été intégré dans un processeur de textes, nous avons procédé à la mise en évidence de chaque mot. Un logiciel appelé Simple Concordance permet d'isoler toute chaîne de caractères allant d'un blanc à un autre, chaque chaîne étant isolée et étiquetée. Le programme indique pour chaque séquence dans quel texte elle apparait, combien de fois et à quel endroit du texte. Nous avons donc appliqué la procédure aux 150 textes. Tout n'a pu être fait de façon automatique, certains signes graphiques n'ayant pu être discriminés. Il a fallu avant chaque texte dresser la liste de tous les signes graphiques utilisés par les auteurs. Nous avons ensuite réuni les documents ainsi traités sous le format txt. C'est à partir de ces documents que nous avons poursuivi le traitement du corpus.

\subsubsection{Introduction des textes dans une base de données}

Une fois les textes traités, chaque mot-texte a été introduit dans une base, chaque mot étant codé avec le texte auquel il appartient. Cette opération, assez longue, a soulevé quelques difficultés, notamment la coupure ou mécoupure des mots. En effet, nous avons conservé dans le corpus informatisé les caractéristiques des mots telles que données par les manuscrits. Cependant, si la table des caractéristiques du corpus original a été conservée dans la base, une autre table a été élaborée dans laquelle les «erreurs» ont été lissées pour faciliter la lecture et le regroupement des mots-texte à notre avis semblables. Le tout a fourni une base comprenant 59902 registres représentant le total des mots composant le corpus original. À partir de ces registres, nous avons obtenu 12499 items. Bien que le texte original de la parabole soit de dimensions réduites, la base de données constitue un ensemble qui permet une série de traitements significatifs. 


\subsection{Lemmatisations}

Notre intention étant celle de procéder à une étude géolinguistique des parlers du Pays basque nord, nous avons eu recours à une lemmatisation, étape indispensable dans le traitement de tout corpus linguistique. Sans lemmatisation, en effet, le corpus est lourd et d'utilisation pénible. Nous avons construit deux lemmatisations, l'une orthographique - relative à la forme des mots et à leurs variantes -, l'autre sémantique ou onomasiologique - relative au signifié.

\subsubsection{Lemmatisation orthographique}

Nous avons décidé de créer un programme auxiliaire pour la lemmatisation (les logiciels existants ne pouvant nous fournir de solution satisfaisante) qui donne tout d'abord la liste des mots à lemmatiser (voir fig. 1).

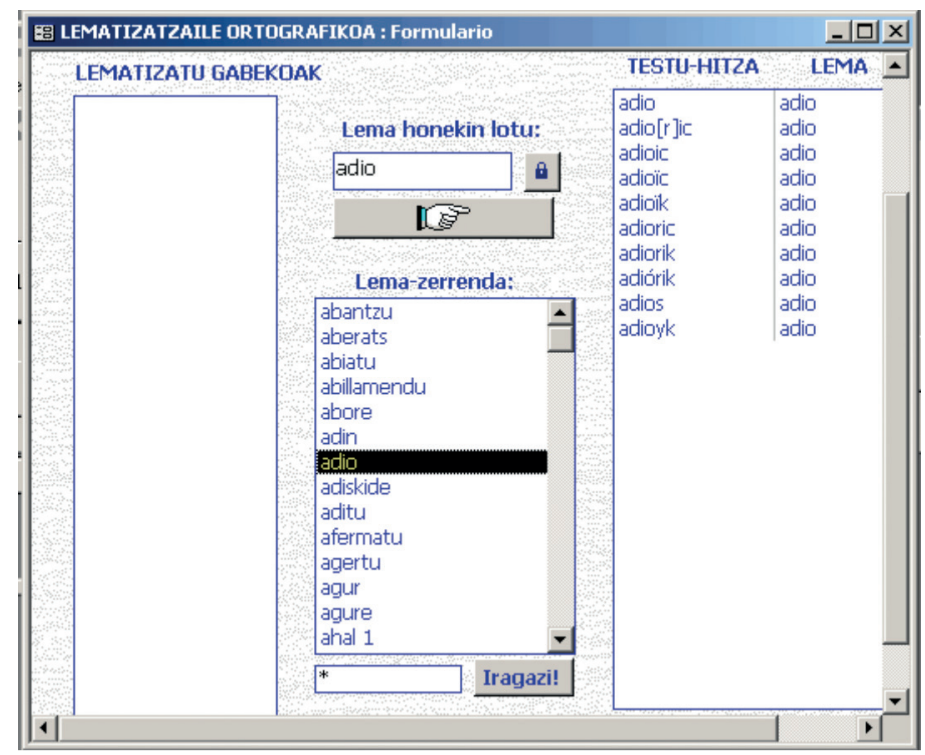

Figure 1. - Écran du programme de lemmatisation.

Les mots-texte sont donnés dans la colonne de gauche dite «lematizatu gabekoak / non lemmatisés». Le chercheur choisit un des mots du texte et lui assigne le lemme qui lui correspond dans le cartouche intitulé «lema honekin lotu / attacher à ce lemme». Ensuite, après avoir sélectionné par un bouton le lemme voulu, les deux termes mis en relation apparaissent dans le cartouche de droite, avec, à gauche, le mot du texte (adio, adio[r]ic, adioic) et, à droite, le lemme choisi (adio). La classification se fait jusqu'à épuisement de la liste des mots. Une vérification ultérieure est possible pour 
s'assurer de la classification adéquate de tous les mots du texte par lemme et il est possible de changer le lemme correspondant à un mot donné du texte. Au milieu de l'écran, se situe la colonne intilulée «lema-zerrenda/ liste des lemmes». Il suffit de sélectionner l'un des lemmes de la liste pour voir dans la colonne de droite la liste des mots-texte classifiés sous ce lemme. Dans cet exemple précis dix mots-texte ont été classifiés sous le lemme adio. Les mots comme adioric portant une marque grammaticale (cas partitif ici) peuvent être ainsi classés avec les formes nues. La «lemmatisation orthographique» permet de fournir le lexique de tout le corpus. Dans ce lexique, chaque lemme constitue une entrée indiquant son nombre d'occurrences, ses variantes et le code de chaque commune dans laquelle se trouve chaque variante. Il s'agit donc d'une procédure lexicographique qui ramène bon nombre d'items différents à des lemmes considérés comme entrées d'un dictionnaire. Au total, nous sommes passés de 12499 items à 1199 lemmes distincts servant d'entrées de dictionnaire.

\subsubsection{Lemmatisation onomasiologique}

Après cette lemmatisation consacrée aux variantes orthographiques ou grammaticales, il fallait procéder à la lemmatisation sémantique. Nous sommes partis du texte source, soit la version française de la Parabole de l'Enfant prodigue. Nous y avons donc distingué un certain nombre de signifiés, au nombre de 151. Nous n'avons pas utilisé tous les signifiés possibles, mais seulement ceux dont la distribution nous intéressait et pour chaque signifié, nous avons examiné comment le terme français avait été traduit par un signifiant basque trouvé dans la liste de la lemmatisation orthographique indiquée ci-dessous. Ici encore, les difficultés n'ont pas manqué. Il nous a fallu faire des choix ou procéder à des exclusions qui peuvent être discutés ou critiqués. Par exemple, pour le signifiant «tomber» les lemmes suivants sont apparus dans la liste : erori, majoritaire avec 97 occurrences, eori avec 24 occurrences offrant la chute de la vibrante intervocalique, mais aussi d'autres termes minoritaires comme jarri, etzatu, paratu, iraili et aurtiki, termes fort éloignés en eux-mêmes de la notion de «tomber». Comme le contexte du texte était «il se laissa tomber sur un escabeau», les solutions des divers traducteurs ont été très variées et pas toujours facilement comparables. De même, un terme comme «traiter» dans le contexte «traitez-moi comme le dernier de vos valets» a fourni maintes solutions comme tratatu, emprunt manifeste qui apparaît 106 fois, ibili et son factitif erabili, six fois chacun, atxiki deux fois, etc. Dans d'autres cas heureusement, et le plus souvent, la surface sémantique du signifié est nettement limitée et la lemmatisation est aisée : «canard», «chemin», «naître»... Cette lemmatisation a bien entendu conditionné dans une grande mesure 
les étapes suivantes du travail. D'une part, elle a supposé un classement lexicographique, d'autre part, elle est marquée par le fait même que l'on travaille à partir d'une traduction. Cette difficulté est bien connue des atlas linguistiques dans lesquels les artefacts provenant de la traduction sont monnaie courante. Nous pouvons donc dire que nous avons ainsi constitué plusieurs bases de données :

a) une base organisée sur une lemmatisation orthographique qui rassemble toutes les données premières, dans laquelle sont réunis tous les mots du texte et toutes les unités grammaticales;

b) une base organisée sur la dite lemmatisation sémantique et qui fournit, prêtes à l'analyse, les données d'ordre lexical ;

c) une base grammaticale réunissant les données phonologiques, morphonologiques et syntaxiques serait à ajouter, mais c'est une base que nous n'avons pas encore réalisée.

Le traitement dialectologique des textes passe par la construction de ces bases de données. L'information distribuée dans le corpus est ainsi organisée pour l'analyse de manière à ce que le chercheur puisse en faire l'étude. Dans les lignes suivantes nous procédons à l'analyse du lexique pour produire une cartographie. Sur les 227 signifiants choisis au départ, certains d'entre eux n'étaient pas traduits. Cela nous a poussé à en éliminer un certain nombre. Les signifiants trop ténus ou trop vastes, ou les réponses rendues trop fragmentées par des choix grammaticaux très éclatés ont été mis en marge. Comme indiqué plus haut, finalement, nous nous sommes limités à un total de 151 termes dont voici la liste alphabétique :

$\begin{array}{llllllll}1 & \text { aimer } & 18 & \text { bruit } & 35 & \text { doigt } & 52 & \text { fils } \\ 2 & \text { aîné } & 19 & \text { canard } & 36 & \text { domestique } & 53 & \text { fleur } \\ 3 & \text { âne } & 20 & \text { champ } & 37 & \text { donner } & 54 & \text { fou } \\ 4 & \text { anneau } & 21 & \text { chanter } & 38 & \text { dormir } & 55 & \text { frère } \\ 5 & \text { appeler } & 22 & \text { chasse } & 39 & \text { égal } & 56 & \text { froid } \\ 6 & \text { apporter } & 23 & \text { chauffer } & 40 & \text { enfant } & 57 & \text { fromage } \\ 7 & \text { après } & 24 & \text { chemin } & 41 & \text { enfin } & 58 & \text { fruit } \\ 8 & \text { argent } & 25 & \text { chien } & 42 & \text { envoyer } & 59 & \text { garder } \\ 9 & \text { arroser } & 26 & \text { chou } & 43 & \text { escabeau } & 60 & \text { gens } \\ 10 & \text { aujourd'hui } & 27 & \text { ciel } & 44 & \text { étoile } & 61 & \text { grand } \\ 11 & \text { avant } & 28 & \text { coq } & 45 & \text { faim } & 62 & \text { hardes } \\ 12 & \text { baiser } & 29 & \text { cou } & 46 & \text { faire } & 63 & \text { heureux } \\ 13 & \text { bœuf } & 30 & \text { coupable } & 47 & \text { femme } & 64 & \text { hier } \\ 14 & \text { boire } & 31 & \text { croire } & 48 & \text { fenêtre } & 65 & \text { homme } \\ 15 & \text { bois } & 32 & \text { dépenser } & 49 & \text { fête } & 66 & \text { ici } \\ 16 & \text { bon } & 33 & \text { dernier } & 50 & \text { feu } & 67 & \text { jambe } \\ 17 & \text { bras } & 34 & \text { dire } & 51 & \text { feuille } & 68 & \text { jardin }\end{array}$




\begin{tabular}{|c|c|c|c|c|c|c|}
\hline jeune & 90 & neuf & 111 & prendre & 132 & tomber \\
\hline joie & 91 & nuit & 112 & prodigue & 133 & tôt \\
\hline jolie & 92 & obeir & 113 & punir & 134 & traîner \\
\hline jurant & 93 & œil & 114 & quitter & 135 & traiter \\
\hline lande & 94 & œuf & 115 & raisin & 136 & traverser \\
\hline larme & 95 & oiseau & 116 & rasembler & 137 & trouver \\
\hline légèrement & 96 & ouvrir & 117 & regarder & 138 & tuer \\
\hline lever (se) & 97 & pain & 118 & réjouir (se) & 139 & veau \\
\hline 77 & 98 & paraître & 119 & répondre & 140 & vendre \\
\hline loin & 99 & pardonner & 120 & reproche & 141 & venir \\
\hline lune & 100 & partager & 121 & retrouver & 142 & ventre \\
\hline maintenant & 101 & partie & 122 & rivière & 143 & veste \\
\hline maison & 102 & pauvre & 123 & s'en aller & 144 & vide \\
\hline maître & 103 & péché & 124 & savoir & 145 & vin \\
\hline malheureux & 104 & père & 125 & soir & 146 & vite \\
\hline manger & 105 & pied & 126 & soulier & 147 & vivre \\
\hline méchant & 106 & pleine & 127 & sueur & 148 & voir \\
\hline mettre & 107 & pommier & 128 & table & 149 & voisin \\
\hline mois & 108 & porc & 129 & temps & 150 & voler \\
\hline naître & 109 & pourrir & 130 & tendre & 151 & vouloir \\
\hline nappe & 110 & poussière & 131 & tiroir & & \\
\hline
\end{tabular}

Nous avons aussi procédé à une réduction du nombre de textes quand une commune a fourni plus d'une version. De 150 textes, nous sommes descendus à 137 puisque les communes suivantes présentaient deux versions : Saint-Jean-de-Luz, Ustaritz, Espelette, Mauléon, Saint-Palais, ArberatsSillègue, Béguios, Iholdy, Ostabat, Saint-Étienne-de-Baïgorry, Saint-JeanPied-de-Port et Tardets-Sorholuz. Ce choix nous a été imposé par l'outil informatique qui n'admet qu'une réponse par commune. La base lexicale, touchant 151 signifiés dans 137 villages, nous a fourni donc un ensemble de 20838 items ou taxats pour reprendre la nomenclature de H. Goebls. Ce nombre nous amène à formuler une remarque importante : tiré d'un texte assez court, ce total de 151 signifiants représente le total le plus élevé qui ait jamais été utilisé à ce jour en dialectologie basque pour opérer un traitement géolinguistique. De plus, il est appliqué seulement à une partie du Pays basque et non pas à tout le domaine de la langue. Ces analyses sont premières et devront être reprises et améliorées afin de fournir rapidement des résultats encore plus fiables. Nous ne sommes pas les premiers cependant à avoir travaillé sur le corpus Bourciez et nous avons cité déjà Jacques Allières (1978) et son «Petit atlas linguistique basque français, Bourciez $1 »$. Ce document présentant 20 cartes commentées, avait l'immense mérite, après Vinson, de signaler l'intérêt pour les dialectologues des textes recueillis par Bourciez. Dans le traitement géolinguistique que nous proposons trois axes de travail peuvent être ouverts : 
- des études basées sur les points de vue habituels et traditionnels de la dialectologie;

— un traitement géolinguistique proche des méthodes de J. Allières;

- un traitement dialectométrique selon les perspectives de H. Goebl.

\section{Cartes lexicales}

Nous avons d'abord procédé à un traitement géolinguistique inspiré des atlas linguistiques traditionnels en publiant des cartes signifié par signifié. Dans le premier volume d'analyse nous avons publié 96 cartes. La raison de ce nombre limité est donnée plus loin. Sur les 151 signifiés que nous avions tirés de la Parabole, nous avons constaté que dans 48 cas la carte obtenue aurait été mononyme : autrement dit la carte ne montrerait aucune variation puisqu'à un seul signifiant français correspond un seul lexème en basque. Quand, de façon très minoritaire, dans quelques communes, on trouve une réponse divergente due à une traduction éloignée, telle que le fait de traduire «doigt» par $e s k u$ «main», nous avons considéré que la carte était mononyme si esku est la seule réponse partout ailleurs. Nous avons aussi neutralisé les marques morphologiques et considéré que, par exemple, gazteago litt. «plus jeune» ne se distinguait pas de gazte «jeune». Quand la différence est morphologique nous avons repoussé l'analyse au troisième volume de notre trilogie. Les termes qui n'ont pas fait l'objet d'une cartographie publiée sont :

\begin{tabular}{|c|c|c|}
\hline âne (3) asto & 20 fête (49) : festa & 39 neufs (90) : berri \\
\hline anneau (4) : eraztun & 21 feu (50): su, egur & œil (93) : begi \\
\hline bœuf (13) : idi & 22 feuille (51): hosto & 41 œuf (94) : arrautza \\
\hline boire (14) : edan & 23 fils (52) : seme & 42 pain (97) : ogi \\
\hline bois (15) : oihan & 24 frère (55): anaia & 43 paraître (98) : agertu \\
\hline bon $(16)$ : on & 25 froid (56) : hotz & 44 pardonner (99) : \\
\hline bras (17) : beso & 26 fromage (57) : gasna & barkatu, barkamendu \\
\hline chauffer (23) : berotu & 27 grand (61): handi & 45 père $(104)$ : aita \\
\hline chou (26) : aza & 28 hier (64) : atzo & 46 pleine (106) : bete \\
\hline $\operatorname{ciel}(27)$ : zeru & 29 homme (65) : gizon, aita & 47 poussière $(110)$ : errauts \\
\hline $\operatorname{coq}(28)$ : oilar & 30 ici (66) : hemen & 48 savoir (124) : jakin \\
\hline cou (29) : beso & 31 jardin (68) : baratz & 49 sueur (127) : izerdi \\
\hline dire (34) : erran & 32 jeune (69) : gazte, gazteago & 50 table (128) : mahai \\
\hline doigt (35): eri, esku & 33 larme (74) : nigar & 51 vendre (140): saldu \\
\hline donner (37) : eman & 34 loin (78) : urrun, urrundu & 52 vide (144) : huts, hustu \\
\hline enfant (40) : haur & 35 maintenant (80): orai & 53 vivre (147) : bizi \\
\hline étoile (44) : izar & 36 maison $(81)$ : etxe & 54 voir (148) : ikusi \\
\hline faire (46) : egin & 37 manger (84) : jan & 55 voisin (149) : auzo \\
\hline fenêtre (48) : leiho & 38 mois (87) : hilabete & 56 vouloir (151) : nahi \\
\hline
\end{tabular}




\subsection{Cartes effectivement réalisées}

Nous avons classé les 96 cartes dans l'ordre alphabétique à partir du français et non pas dans l'ordre d'apparition dans le texte, pour des raisons de commodité. Pour chaque carte nous donnons le nom du signifiant français avec son contexte, puis :

a) la liste des mots-texte, par commune et en conservant l'orthographe originale tirée du manuscrit ;

b) la carte elle-même, en couleurs, bâtie sur les polygones de Thiessen, le titre de la carte reprenant le signifiant français;

c) la légende fournit la liste des lemmes sous lesquels ont été classées les réponses du texte. À chaque lemme est assignée une couleur dont on trouve le répondant sur la carte;

d) les observations nécessaires à la compréhension de certains choix de cartographie ou de lemmatisation.

Le fond de carte reprend le territoire de Soule, Basse-Navarre et Labourd, mais nous n'avons pas porté les communes dans lesquelles Bourciez n'a pas recueilli de version basque. Le fond de carte est le même dans tout le travail, construit sur 138 communes dont chacune est représenté par un polygone. Il va de soi que ce polygone ne suit pas les limites géographiques réelles de chaque commune sur le terrain. Les cartes de ce type ont une tradition bien connue en géolinguistique, surtout depuis les travaux fondateurs de Hans Goebl. L'Atlas linguistique du Pays basque sur lequel nous travaillons au sein d'Euskaltzaindia repose sur la même cartographie (dans cet atlas, toutes les communes bascophones n'ont pas été enquêtées). Pour le tracé des polygones, on a suivi la méthode inaugurée par Voronoï et Thiessen. Hans Goebl donne toute information sur ce mode de représentation (Goebl, 1981 et 1992). Que l'on sache cependant que dans un tel polygone, tout point appartenant à ce polygone est plus près du centre de son polygone que du centre de tout autre polygone. Sur la carte, chaque polygone porte un numéro, qui est celui de la commune dont la liste apparaît sur la page de gauche de notre ouvrage. Ceci permet d'identifier assez aisément chaque commune, la taille de chaque polygone étant cependant variable, plus grande en Haute-Soule qu'autour de Saint-Palais ou SaintJean-Pied-de-Port. Voici la liste des communes du recueil Bourciez ayant fourni une version en langue basque : 
1193-Lehuntze / Lahonce

1194-Mugerre / Mouguerre

1196-Urketa / Urcuit

1198-Arrangoitze / Arcangues

1201-Donibane-Lohizune / Saint-Jean-de-Luz

1202-Donibane-Lohizune / Saint-Jean-de-Luz

1203-Azkaine / Ascain

1204-Bidarte / Bidart

1205-Biriatu / Biriatou

1206-Ziburu / Ciboure

1207-Getaria / Guéthary

1208-Hendaia / Hendaye

1209-Urruña / Urrugne

1210-Uztaritze / Ustaritz

1211-Uztaritze / Ustaritz

1212-Ahetze / Ahetze

1213-Arbona / Arbonne

1214-Haltsu / Halsou

1215-Jatsu / Jatxou

1216-Larresoro / Larressore

1217-Senpere / Saint-Pée-sur-Nivelle

1218-Milafanga / Villefranque

1219-Ezpeleta / Espelette

1220-Ezpeleta / Espelette

1221-Ainhoa / Ainhoa

1222-Kanbo / Cambo

1223-Itsasu / Itxassou

1224-Luhuso / Louhossoa

1225-Sara / Sare

1226-Zuraide / Souraïde

1227-Hazparne / Hasparren

1228-Lekuine / Bonloc

1229-Makea / Macaye

1230-Mehaine / Méharin

1231-Lekorne / Mendionde

1232-Donostiri / Saint-Esteben

1233-Donamartiri / Saint-Martin

1236-Aiherra / Ayherre

1237-Beskoitze / Briscous

1238-Bastida / Labastide-Clairence

1243-Bardoze / Bardos

1249-Maule / Mauléon

1250-Maule / Mauléon

1251-Ainharbe / Ainharp

1252-Ürrüstoi-Larrabile / Arrast-Larrebieu

1253-Altzürükü / Aussurucq

1254-Barkoxe / Barcus

1255-Sarrikotapea / Charritte-de-Bas
1256-Sohüta / Chéraute

1257-Ündüreine / Espès-Undurein

1258-Garindaine / Garindein

1259-Gotaine-Irabarne / Gotein-Libarrenx

1260-Ospitalepea / L'Hôpital-Saint-Blaise

1261-Idauze-Mendi / Idaux-Mendy

1262-Mendikota / Menditte

1263-Mitikile / Moncayolle

1264-Muskildi / Musculdy

1265-Urdiñarbe / Ordiarp

1266-Arrokiaga / Roquiague

1267-Bildoze / Viodos

1268-Donapaleu / Saint-Palais

1269-Donapaleu / Saint-Palais

1270-Aiziritze / Aicirits

1271-Amenduze / Amendeuix

1272-Amorotze / Amorots

1273-Arberatze-Zilhekoa / Arbérats-Sillègue

1274-Arberatze-Zilhekoa / Arbérats-Sillègue

1275-Arboti-Zohota / Arbouet-Sussaute

1276-Arüe / Aroue

1277-Arruta-Sarrikota / Arraute-Charritte

1278-Behauze / Béguios

1279-Behauze / Béguios

1280-Behaskane-Laphizketa /

Béhasque-Lapiste

1281-Bithiriñe / Beyrie-sur-Joyeuse

1282-Gamue-Zohazti / Camou-Suhast

1283-Domintxaine-Berroeta /

Domezain-Berraute

1284-Etxarri / Etcharry

1285-Gabadi / Gabat

1286-Garruze / Garris

1288-Ilharre / Ilharre

1290-Larribarre / Larribar

1291-Lohitzüne / Lohitzun

1292-Lukuze-Altzumarta /

Luxe-Sumberraute

1293-Martxuta / Masparraute

1294-Oragarre / Orègue

1295-Ostankoa / Orsanco

1297-Pagola / Pagolle

1298-Uharte-hiri / Uhart-Mixe

1299-Iholdi / Iholdy

1300-Iholdi / Iholdy

1301-Arhantsusi / Arhansus

1302-Armendaritze / Armendarits

1303-Bunuze / Bunus 


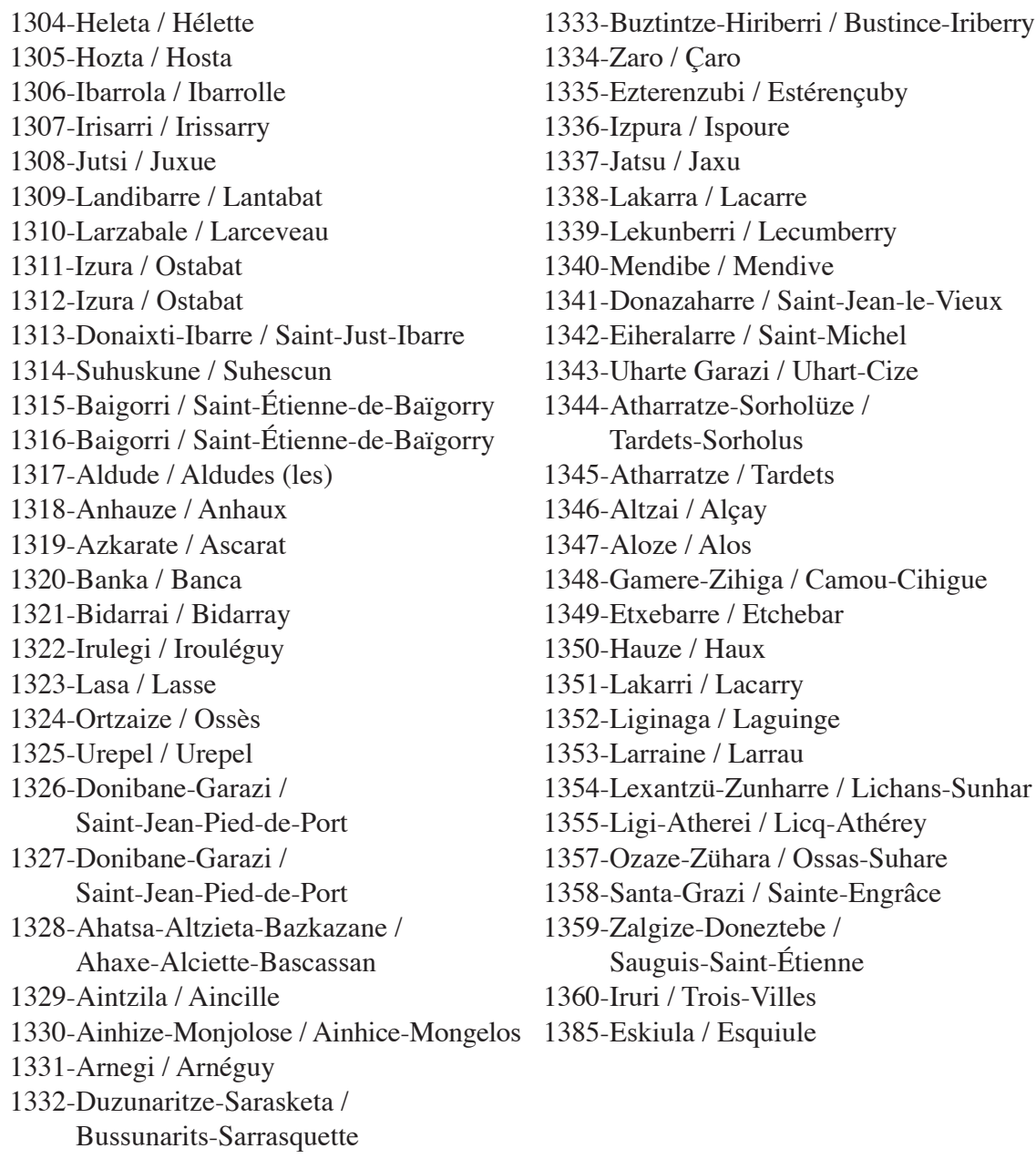

\subsubsection{Les légendes}

Chaque carte a sa légende construite d'après la lemmatisation, elle fournit la liste des lemmes dans lesquels toutes les réponses des textes ont été distribuées. La couleur de chaque lemme se retrouve sur la carte. Il faut ajouter quelques remarques sur la légende et donc la lemmatisation :

a) il arrive que quelques lemmes ne correspondent pas à une traduction exacte du signifiant français. Dans la carte «ressembler (se)», des réponses comme heldu et jin (qui signifient «venir») peuvent apparaître incompréhensibles en dehors du contexte. Il faut se reporter au texte lui-même qui éclaire sur ces termes imprécis; 
b) il existe des signifiés pour lesquels certaines traductions n'offrent aucun équivalent. Nous nous retrouvons dans la situation lacunaire bien connue en géolinguistique, celle des réponses zéro. On leur assigne la dernière position dans les lemmes de la légende et aucune couleur - sinon le blanc - n'est donnée sur la carte pour ces réponses zéro;

c) chaque lemme est suivi d'un nombre qui indique le nombre de parlers ou versions dans lequel il apparaît. Il donne déjà en soi une idée de la distribution géolinguistique;

d) le chiffre qui précède le lemme indique l'ordre dans lequel ces lemmes sont donnés, mais il n'a aucun intérêt géolinguistique.

Cependant, nous avons tenté de regrouper les lemmes en allant de ceux ayant le plus d'occurrences (espace étendu) vers ceux les moins représentés (aire exiguë).

\subsection{Liste des mots-texte}

L'information donnée sur la carte n'est pas toujours exactement la même que la liste des formes attestées dans les textes. On a vu que les formes du corpus n'entrent dans la carte qu'après avoir été lemmatisées; aussi, sur la page de gauche, en face de la carte correspondante, se trouve la liste des formes attestées dans les documents. Ces formes sont données dans la graphie du texte du manuscrit. Sur cette page on retrouve donc :

- le titre de la carte avec la phrase dans laquelle se trouve le mot traduit (en italique);

- le code du texte et de la commune;

- le nom de la commune en basque;

- le mot du texte dans sa graphie originale.

\subsubsection{La liste des lemmes}

À la suite des cartes, nous avons fourni dans l'ouvrage la liste des lemmes ayant servi à la construction de ces cartes. Les lemmes sont donnés dans l'ordre alphabétique et chacun d'eux est suivi du numéro de la carte dans lequel il apparaît. S'il apparaît dans plus d'une carte, le lemme est répété.

\subsubsection{Les cartes grammaticales}

Les cartes grammaticales sont réunies dans le troisième volume consacré aux données Bourciez (Aurrekoetxea, Videgain \& Iglesias, 2008 [2005]). Nous avons lu les textes en essayant d'y trouver une série d'objets lin- 
guistiques intéressant la morphosyntaxe, tout en y ajoutant des questions d'ordre phonologique. Le texte étant d'une longueur limitée, bien des objets linguistiques sont restés en dehors du champ de cette étude. Nous avons organisé ces 101 objets linguistiques de la façon suivante :

a) les cartes 1 à 11 sont consacrées à la déclinaison (génitif, sociatif, ablatif, datif pluriel, partitif) à partir des exemples proposés par le corpus ;

b) dans le groupe des cartes $12-28$, nous avons réuni essentiellement déterminants, pronoms et adverbes de lieu (possessif, démonstratif, indéfini...);

c) le groupe des cartes 29-57 est consacré à la morphologie verbale, depuis le verbe izan, «être» monovalent jusqu'aux formes tripersonnelles et allocutives;

d) un groupe consacré à «plus», «très», «même», «car», sans grande unité (58-61);

e) le groupe 62-75 réunit les questions de syntaxe, surtout les propositions subordonnées;

f) enfin le dernier groupe (75-101) réunit les cartes phonologiques. Bien entendu, elles fournissent aussi une information lexicale dans ces cartes monolexématiques dans laquelle la seule variation est phonologique, comme pour les deux seules variantes zeru ou zelü pour «ciel».

\subsubsection{Méthodologie}

Nous avons suivi la même méthodologie que pour les cartes lexicales, mais la lemmatisation a été faite selon d'autres critères. Nous y reviendrons en une autre occasion.

\section{De la base de données aux matrices de similarité et matrices de distance : la dialectométrie}

La base de données utilisée nous a permis d'étudier la distribution géolinguistique de divers objets linguistiques de manière qualitative. La nature même d'une telle base de données la rend peu pratique à utiliser telle quelle pour recourir à des procédures statistiques classificatoires. Aussi, nous nous sommes proposé de transformer cette première base de données en une base de données numérique en réalisant les opérations suivantes :

- assigner un chiffre à chaque lemme ou taxat pour chaque signifié; 
- attribuer à chaque taxe ou lemme donné qui apparaît dans une commune le code qui correspond à cette commune.

Pour cette procédure statistico-classificatoire nous avons utilisé le logiciel VDM développé par le professeur H. Goebl à l'université de Salzbourg. Cette décision survient après d'autres réflexions dans le domaine et repose sur des raisons précises. Une première réflexion nous a fait éliminer le traitement manuel des données. On sait que parmi les précurseurs de la dialectologie il faut citer Jean Séguy, professeur à l'université de Toulouse, auteur de l'Atlas linguistique et ethnographique de la Gascogne, qui ne disposait pas encore des ressources de l'informatique et de la statistique. Gotzon Aurrekoetxea a commencé par étudier la structuration géolinguistique des dialectes basques en Navarre, puis celle des parlers biscayens, avant d'aborder d'autres travaux. Nous prolongeons ici cette réflexion. Ces travaux partent de la conviction qu'une des conditions de la géographie linguistique pour progresser et se moderniser est de se munir d'outils qui lui permettent de sortir de l'atomisme. Il faut donc nous efforcer d'avancer dans les théories du changement linguistique, ne serait-ce que sur des cantons bien délimités.

Un certain nombre d'atlas linguistiques sont des musées dans lesquels une énorme documentation a été réunie, souvent de manière précise et exemplaire, mais qui dort sans qu'on en tire toutes les matières souhaitables; au contraire, elle doit être rendue consultable et utilisable pour nos analyses. L'orientation principale de ces analyses veut donc être une synthèse des richesses observées et recueillies dans les atlas. Dès les premiers travaux de dialectologie, chez Jean Séguy et davantage chez Hans Goebl, est revendiquée la nécessité d'utiliser des procédures statistico-classificatoires appliquées en linguistique et dialectologie de la même manière qu'elles sont utilisées dans d'autres sciences en général. Dans ce parcours, trop souvent les instruments de classification se sont révélés d'un emploi difficile, car ils n'étaient nullement destinés à des recherches linguistiques ni dessinés dans cet objectif.

L'aide de personnes étrangères au champ de la linguistique a été indispensable et bien du temps a été passé au maniement d'instruments compliqués. Nous tentons désormais de nous équiper, en tant que dialectologues, de l'environnement informatique qui nous permettra d'étudier la classification géolinguistique des parlers et d'être autonomes. De ce point de vue le programme VDM mis au point par H. Goebl est extrêmement puissant et de maniement aisé; d'une part, il possède les avantages d'un outil statisticoclassificatoire, d'autre part il est capable d'une cartographie automatisée. Il est donc orienté vers les besoins des linguistes et dialectologues, le tout 
réuni en un seul instrument. Cependant, on ne peut en tirer profit sans dominer les concepts de base de la statistique et des méthodes classificatoires : le dialectologue doit donc suivre une bonne formation de base afin de comprendre quels sont les concepts qu'il est amené à utiliser pour en tirer les résultats souhaitables. Hans Goebl a ainsi à maintes reprises insisté sur l'importance des concepts de similarité et de matrice des distances (Goebl, 1992). Le chemin de l'une à l'autre se fait par l'indice de similarité. Les taxonomies nouvelles présentent bon nombre d'indices comparables à celui-là (Sneath \& Sokal, 1973; Chandon \& Pinson, 1981; Bailey, 1994; Gordon, 1999). Le dialectologue doit choisir parmi ces indices en fonction de ses besoins et de ses objectifs. Suivant H. Goebl de près, nous avons choisi «l'indice relatif d'identité» IRI (RIW en allemand) et «l'indice pondéré d'identité» IPI (GIW en allemand). Ces deux indices sont utilisés de longue date par $\mathrm{H}$. Goebl et ont fait la preuve de leur efficacité. Nous reprenons à notre compte la citation de Goebl qui explique la différence entre les deux indices :

Alors que l'RIW ${ }_{\mathrm{jk}}$ est un indice isocratique (ou «adansonien», baptisé d'après le naturaliste français Michel Adanson, 1727-1806) et traite ainsi tous les taxats de la matrice de données numériquement sur un pied d'égalité, l'IPI $(1)_{\mathrm{jk}}$ confère, comme indice anisocratique, un poids numérique plus grand aux taxats géographiquement moins répandus tout en défavorisant les taxats de grande diffusion spatiale. L'action pondératrice de cet indice traduit en termes taxométriques une opinion très habituelle parmi les linguistes selon laquelle les traits linguistiques universellement répandus disposeraient d'une importance classificatoire moindre que les traits linguistiques moins fréquents auxquels on attribue souvent la prérogative d'être ou bien des archaïsmes ou bien des innovations dignes d'un intérêt majeur. (2003: 69)

Il est bien clair donc que si l'indice RIW traite de la même manière tous les taxats, l'indice IPI donne un poids plus élevé aux taxats de diffusion réduite sur l'espace. Nous utiliserons donc ces deux indices. Avec l'indice de similarité, nous avons recours à l'indice de distance (on utilise aussi l'indice de dissimilarité ou différenciation), aussi bien l'indice de distance relatif dit $\operatorname{IRD}_{\mathrm{jk}}$ que l'indice de distance pondéré dit $\operatorname{IRD}(1)_{\mathrm{jk}}$. On les utilise en appliquant la formule suivante : $I D_{j k}=100-I D_{j k}$ pour l'indice relatif ou bien $\operatorname{IRD}(1)_{\mathrm{jk}}=100-\operatorname{IRD}(1)_{\mathrm{jk}}$ pour l'indice pondéré. De cette manière on mesurera les distances linguistiques. Ces deux indices, similarité et distance, sont des outils pour mesurer de manière mathématique et précise la fonction communicative et la fonction distinctive entre les parlers. Ces concepts de communication et distinction étant fort utilisés dans la dialectologie «théorique», les deux outils cités ici sont donc importants. 


\subsection{Les algorithmes de regroupement}

L'algorithme de groupement est un outil qui permet de regrouper et d'échelonner la différence entre les parlers que la matrice de similarité met en évidence. Ce regroupement et cet échelonnement sont nécessaires pour montrer et exposer sur la carte les distances linguistiques de manière visible. La similarité ou la distance différente étant impossible à visualiser sur une carte, les données doivent être regroupées avant d'être rendues visibles sur une seule carte. Il existe plus d'un algorithme capable d'effectuer cette tâche : nous en utilisons deux, les algorithmes MINMWMAS et MEDMW. Outre le fait qu'ils soient très connus, ils sont bien adaptés à ce que nous recherchons.

\subsubsection{L'algorithme MINMWMAS (en six groupes)}

Nous résumons ici les explications données en détail par Goebl (1981 : $364 ; 1987$ : 79 et suiv.). Nous prenons pour point de départ un point zéro qui représente un parler donné, celui d'une commune donnée et à partir de cette commune nous examinons les relations qu'elle a avec les autres communes, autrement dit nous examinons la distance linguistique entre cette commune donnée et chacune des autres, en tenant compte des caractéristiques suivantes :

a) on calcule d'abord la moyenne arithmétique des distributions des fréquences;

b) on divise par trois la distance qui sépare la fréquence la plus petite de la moyenne arithmétique; le nombre obtenu sera la longueur de chaque groupe. En commençant par la fréquence la plus faible on obtient trois groupes inférieurs à la moyenne;

c) on opère de la même façon avec les fréquences supérieures à la moyenne arithmétique; on obtient trois groupes supérieurs à la moyenne.

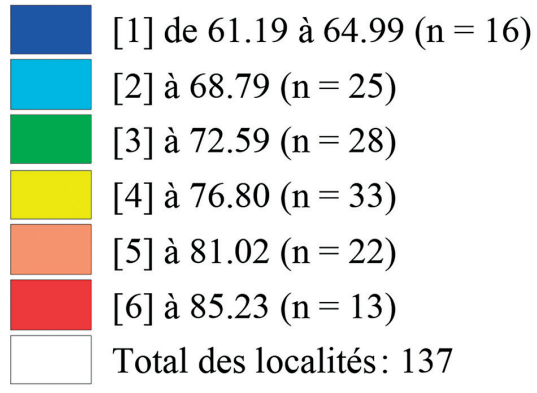

Figure 2. - Un exemple d'algorithme MINMWMAX. 
Dans cet exemple la moyenne arithmétique est 72,59 et la fréquence la plus faible 61,19. La différence entre les deux est donc de 11,4 qui, divisée en trois groupes donne le nombre 3,8 qui nous servira à établir les trois groupes inférieurs, soit $61,19+3,8=64,99$ ( $1^{\text {er }}$ groupe $) ; 64,99+3,8=$ 67,79 (groupe 2) et enfin 68,79+3,9=72,59 (groupe 3). Dans les groupes supérieurs on opère de la même manière et la distance avec la moyenne la plus forte est 12,64 , qui, divisée par 3, donne 4,21 et permet d'établir le groupe 4 (allant jusqu'à 76,80), le groupe 5 (allant jusqu'à 81,02 ) et le groupe 6 (allant jusqu'à 85,23).

\subsubsection{L'algorithme MEDMW (en six groupes)}

Cet algorithme est moins «neutre» que le précédent, car il force davantage les données en diminuant les poids des données médianes et en augmentant le poids des données extrêmes (Goebl, 1987 : 81-82). La procédure de cet algorithme est la suivante :

a) on ordonne la distribution des fréquences de la plus faible à la plus forte;

b) on choisit la fréquence la plus proche de la moyenne arithmétique;

c) on réunit en un groupe les valeurs des fréquences allant de la plus faible à celle la plus proche de la moyenne arithmétique;

d) on réunit aussi dans un groupe les valeurs des fréquences en partant de la plus haute;

e) on divise chacun de ces deux groupes en trois sous-groupes, chaque groupe ainsi déterminé ayant le même nombre de distribution de fréquences ou un nombre équivalent.

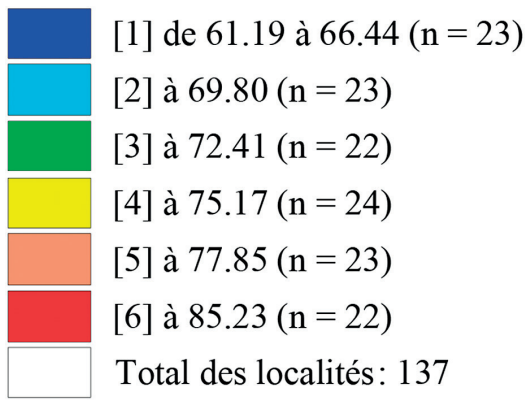

Figure 3. - Exemple de l'algorithme MedMw.

Nous utilisons les mêmes chiffres que dans l'algorithme MINMWMAX. Les données extrêmes sont les mêmes, mais le mode de regroupement 
change ici. On trouve 22 parlers dans les groupes 3 et 6, 23 dans les groupes 2 et 5 ; et 24 parlers dans le groupe 4 .

\subsection{Les histogrammes}

En plus des cartes et des algorithmes, les histogrammes permettent de montrer le poids de chaque groupe de valeurs dans le programme VDM. Les histogrammes complètent les cartes choroplèthes et montrent les caractères statistiques de la distribution de la fréquence. La construction des choroplèthes se fait par un algorithme bien particulier. Dans les histogrammes, on étudie le poids des divers groupes distingués par les algorithmes cités ci-dessus. Plus un groupe donné réunit un nombre élevé de parlers et plus sa hauteur dans l'histogramme sera élevée. Le lecteur peut se rendre compte que chaque groupe fourni par le regroupement calculé par l'algorithme apparaît divisé en deux colonnes dans l'histogramme, et donc, que de ce point de vue, il fournit davantage d'informations.

De plus, l'écart-type calculé par la distribution de la moyenne arithmétique et de la distribution des moyennes de l'histogramme permet par rapport à la courbe normale théorique de contrôler un certain nombre de détails statistiques. Si la courbe est équilibrée, en ayant le même nombre de parlers à droite qu'à gauche, on parle de courbe symétrique. Ce type de courbe montre une caractéristique importante dans les parlers étudiés, qui est que le parler donné a un niveau de relations entre les parlers qui est équilibré. Autrement dit ce parler a autant de parlers ayant de bonnes relations avec lui que de parlers ayant de mauvaises relations. Par contre, si la courbe de l'histogramme est asymétrique à droite, ce parler a plus de parlers qui sont en mauvaises relations que de parlers qui sont en bonnes relations. À contrario si la courbe est asymétrique à gauche. Enfin, chaque colonne de l'histogramme donne le nombre de parlers qu'elle réunit. C'est ainsi que le parler portant le code 1209 (Urrugne) a de bonnes relations avec 70 parlers (excellentes avec 13, très bonnes avec 24 et bonnes avec 35) et de mauvaises relations avec 67 parlers (extrêmement mauvaises avec 18 parlers; très mauvaises avec 26 et mauvaises avec 23 ). On ne verra aucune allusion morale dans l'emploi de «bon» et «mauvais».

\subsection{Cartographie et fonds de carte}

Afin de cartographier les analyses statistico-classificatoires nous utilisons les polygones de Thiessen qui repose sur la triangulation de Delaunay. Ce type de polygonisation n'est pas nouveau en géolinguistique et $\mathrm{H}$. Goebl l'utilise depuis 30 ans. Mais cette méthode était déjà recommandée par le germaniste Carl Haag en 1898 (Goebl, 1981 : 363-364; Goebl, 1987 : 
93-99; Goebl, 2003 : 72-72). Chaque polygone ainsi obtenu correspondant à chaque parler et chaque commune permet d'y inscrire ses caractéristiques, souvent par l'intermédiaire de la couleur. Dans le cas du recueil Bourciez, nous avons calculé le polygone correspondant à chaque point d'enquête. Chaque polygone porte le même code que celui de la commune correspondante. Seules les communes ayant fourni une réponse sont portées sur la carte. Il faut donc toujours garder à l'esprit qu'il existe sur le terrain d'autres communes dont notre cartographie ne tient pas compte. Les cartes élaborées dans ce travail suivent des conventions extrêmement précises telles que la progression des couleurs dans la logique du spectre de l'arc en ciel. Le chercheur peut, s'il le veut, modifier les couleurs, ou le nombre de couleurs (dans les cartes synthétiques). La question des points sans réponse est toujours un écueil en géolinguistique. Trop nombreux, les blancs risquent de distorsionner la carte. Dans notre cas, ils représentent 238 blancs sur 20838 données, soit 1,14\%. Ceci est peu et les conséquences n'en sont pas dramatiques. Cependant on trouvera un total de 97 cartes qui présentent au moins un polygone en blanc. Le «responsable» en est la commune de Hendaye où la traduction est moins bien complète et accuse à elle seule un total de 83 blancs.

\subsection{Les cartes onomasiologiques}

Les premières productions du programme VDM sont des cartes onomasiologiques. On examine par la carte comment les textes répondent à une notion telle que «joie», «pauvre», «porc», etc., comme la carte «bruit» telle qu'elle apparaît en carte 1.

Nous avons choisi de distribuer l'ensemble des réponses au mot «bruit» en 7 lemmes qui sont azantz, harrabots, herots, haro, harramantza, hots et kalapita. Les trois premiers représentent chacun un nombre d'occurrences bien plus important que les quatre derniers. Leur répartition n'est pas non plus stochastique et l'on voit bien que azantz représente une aire centrale (en gros, bas-navarraise) tandis que harrabots signale un espace labourdin mordant sur la Basse-Navarre occidentale, tout en constatant que herots est caractéristique des parlers souletins à l'est. Une couleur, en principe «parlante» permet de discriminer les lemmes. Cette classification est essentielle pour la carte onomasiologique donnée, mais aussi pour un traitement plus synthétique. Nous avons dit que 151 items ont été traités à partir des textes Bourciez, mais aussi que parmi eux 54 items présentaient des cartes mononymes, c'est-à dire sans variation lexicale, soit un tiers. Il ne fait pas de doute que c'est un fait significatif quand on veut étudier la structuration de la réalité géolinguistique dans les trois provinces étudiées. 


\subsection{La classification des parlers}

Depuis la carte de Louis-Lucien Bonaparte et ses multiples essais de classification des dialectes basques, bien d'autres tentatives respectables ont eu lieu pour représenter leur structuration, soit en revisitant les matériaux obtenus, soit en recourant à des méthodologies plus novatrices. Nous l'illustrons ainsi par une série de cartes nouvelles que nous commentons.

\subsection{La distance linguistique entre les parlers ou DM interponctuelle}

La dialectométrie interponctuelle est une branche de la discipline qui sert à mesurer la distance linguistique entre deux communes du territoire examiné. Elle s'intéresse soit à la similarité entre les deux parlers étudiés, soit à leur distance linguistique. Dans une direction, on étudie ce qui réunit les deux parlers, et dans l'autre, ce qui les distingue. Pour ce faire, nous entrons dans une procédure globalisante. Nous n'utilisons pas les données d'une seule carte, d'un seul item sémasiologique dans notre cas, mais nous prenons en compte l'ensemble des données fournies par la base. Les cartes 2 et 3 sont parmi celles qui offrent le moins de complexité. Le traitement statistique y est réduit. On y montre les similarités et différences tirées des données des 151 cartes, en prenant les communes deux à deux.

\subsubsection{Distance linguistique entre deux communes contiguës}

Nous signalons que le terme «isoglosse » n'a pas tout à fait le même sens que dans la dialectologie traditionnelle. En effet, nous pouvons traiter ici d'isoglosse synthétisant, dans une vision globalisante des données. Il ne s'agit pas donc d'un isoglosse concernant un seul objet linguistique, mais une ligne imaginaire qui met en évidence la différence graduelle entre deux parlers en se basant sur une somme de données. Du point de vue technique, cet isoglosse suit les bords des polygones placés de part et d'autre de celui-ci, il est donc matérialisé par une succession de segments rectilignes et non pas par une ligne ondulante suivant la limite géographique des communes concernées comme cela se fait en cartographie traditionnelle. Dans la dialectométrie interponctuelle, on ne traite que les communes contiguës. La deuxième carte montre bien ce que nous voulons dire : elle est réalisée à partir de l'indice de similarité GIW. L'algorithme de regroupement est MINMWMAX, ce qui facilite l'apparition de données réparties en quatre groupes et donc de quatre couleurs sur la carte. Aux couleurs chaudes (rouge et ocre) correspondent les groupes allant de la moyenne faible $(2,65)$ à la moyenne arithmétique $(60,64)$ et aux couleurs rouges les groupes allant de la moyenne arithmétiques à la moyenne forte $(86,55)$. Il faut comprendre que cette carte visualise la relation linguistique qu'entre- 
tient chaque parler. La couleur rouge et ocre signale avec quels parlers un parler donné a une grande relation linguistique ou une similarité assez élevée. Ce type de relation apparaît surtout en Soule et Labourd. Mais aussi en partie en Basse-Navarre : les types de relations faibles ou mauvaises étant plus nombreux. Il est significatif de voir le haut degré de mauvaises relations entre Basse-Navarre et Soule. La frontière de province est très semblable à la distance linguistique. Dans la carte 3 , bien qu'on utilise le même indice de similarité, GIW, l'algorithme de regroupement est différent; il s'agit de MEDMW qui donne aussi quatre groupes. Les données sont les mêmes qu'en carte 2. Les différences visibles sur la carte sont donc à imputer au changement d'algorithme.

Il faut ajouter qu'outre la nature des couleurs chaudes ou froides qui renseignent sur la qualité des relations linguistiques, l'épaisseur du trait qui relie les parlers est significative. Plus le trait est épais, plus la distance linguistique est grande; plus le trait est fin, plus la distance linguistique est réduite. Dans la carte 2, la plupart des parlers qui entretiennent de bonnes relations sont groupés sur deux espaces, le premier très nettement, en Soule, le second, de manière moins précise, en Labourd. Autour de la frontière entre Labourd et Basse-Navarre, la frontière linguistique se détache, en couleurs bleu clair ou bleu sombre. Il n'apparaît pas en Basse-Navarre un espace à forte cohésion : les isoglosses bleu clair ou bleu foncé y sont nombreux, bien qu'on y trouve quelques isoglosses aux couleurs ocre ou rouge. Cette carte signale donc que la Basse-Navarre ne montre pas un territoire linguistique composé de parlers ayant entre eux une très grande proximité ni donc une grande homogénéité.

Des isoglosses de couleur bleue séparent Basse-Navarre et Soule, davantage au sud qu'au nord. Cette rupture est évidente et montre une faille importante entre les deux domaines. Il s'agit d'un phénomène de grande importance géolinguistique. La Soule est l'espace présentant la plus forte proximité linguistique entre ses parlers, et la plus grande homogénéité, sauf à Pagolle dans le recueil Bourciez. Mais l'instituteur de Pagolle a manifestement rédigé le texte dans un parler basque éloigné de celui de la commune, ce qui explique qu'il semble jouer une partition propre dans ce recueil.

La carte 3, obtenue avec l'algorithme MEDMW, montre des espaces linguistiques plus visibles. En effet, cet algorithme augmente les contrastes en rapprochant les proximités et en éloignant les oppositions. Ici encore, c'est la Soule qui est l'espace le plus cohérent avec une multitude d'isoglosses rouges. Avec cet algorithme, la frontière entre Labourd et BasseNavarre est plus marquée, tout comme celle entre Basse-Navarre et Soule. Une zone de transition importante s'étend entre Basse-Navarre et Labourd, 
tandis que la rupture entre la Basse-Navarre et la Soule est très nette, surtout au sud en zone de montagne.

\subsubsection{Carte de similarité : interprétation géolinguistique de la carte 4}

Cette carte basée sur la triangulation de Delaunay montre la fonction communicative des parlers, c'est-à-dire la similarité ou ressemblance entre les parlers. Comme les cartes 2 et 3 cette carte ne compare que des parlers contigus, soit chaque parler avec ses premiers voisins. Cependant, elle nous intéresse du point de vue géolinguistique. Elle sert à détecter les noyaux dialectaux homogènes; plus le trait est épais, et plus il est «chaud» et donc la proximité linguistique est importante. L'un de ces noyaux le plus net est celui visible en Soule qui va jusqu'aux limites du pays de Mixe. Les parlers qui le composent ont de bonnes ou très bonnes relations entre eux. Ils dessinent un espace ferme, à la grande homogénéité. En revanche, en Basse-Navarre, les parlers ayant de bonnes relations ne dessinent pas un espace marqué. On peut en trouver quelques-uns par vallées comme en Ostabarret (1306-Ibarrole, 1308-Juxue, 1310-Larceveau, 1311-Ostabat auxquels s'ajoutent les voisins 1307-Irissarry, 1309-Lantabat. De même, une homogénéité se manifeste entre Les Aldudes, Saint-Étienne-de-Baïgorry, Arnéguy, Uhart-Cize et Lasse. Dans l'ensemble, la Basse-Navarre est avare d'espaces très unis. La situation est assez comparable en Labourd. On notera en Labourd oriental que des parlers ont de très mauvaises relations avec les voisins de Basse-Navarre. Les chiffres sont bien en dessous de la moyenne arithmétique. Parmi les parlers ayant de très mauvaises relations avec les voisins, on retrouve Pagolle (1297), en raison d'une «imposture» de l'instituteur local. Plus curieusement Luxe-Sunberraute (1292) a aussi de mauvaises relations. On y verra aussi, moins nettement qu'à Pagolle, que l'instituteur a eu recours à bon nombre de tours très occidentaux. Reste le cas de Louhossoa (1224) et Amorots (1272) isolés de leurs voisins. Dans l'ensemble, on peut dire qu'à l'exception de la Soule, le territoire examiné ne présente pas un ensemble dialectal très homogène s'il faut en croire le matériau que nous avons utilisé.

\subsubsection{Les cartes choroplèthes : les cartes entre les parlers (cartes 5, 6, 7 et 8)}

Les cartes synoptiques suivantes montrent les relations linguistiques qu'entretient chaque parler avec les autres parlers. On peut ainsi obtenir le profil de chaque parler.

Dans les cartes précédentes (2, 3 et 4$)$, chaque parler était envisagé par rapport à ses proches voisins afin de dégager des noyaux dialectaux plus ou moins homogènes. 
Dans les cartes suivantes, nous franchissons un autre pas géolinguistique. Nous prenons pour point de départ un parler donné et nous examinons quel degré d'éloignement ou de proximité possèdent tous les autres parlers par rapport à lui. Dans ce calcul, ce sont toutes les données - 151 cartes lexicales - qui sont convoquées. Ces cartes sont appelées «cartes de similarité» et sont typiques de la dialectométrie interponctuelle, ou «cartes choroplèthes» dans la terminologie de Hans Goebl. Dans la carte de similarité le point de départ apparaît en blanc, comme Urrugne dans la carte 5. Un algorithme permet de calculer similarités et différences par rapport à ce point en jouant toujours des couleurs chaudes et froides.

Dans la carte 5, sur le point de référence d'Urrugne, les parlers les plus proches forment un groupe de 13 parlers, tous situés en Labourd, sauf Banca. Les autres communes du Labourd font aussi partie des parlers qui sont situés au-dessus de la moyenne arithmétique, sauf Bardos (1243) et Hendaye (celui-ci n'est pas à prendre en compte au même niveau que les autres puisqu'il fournit une traduction libre). En Basse-Navarre et toujours en référence à Urrugne, on trouve deux groupes : l'un composé de couleur ocre (au-dessus de la moyenne arithmétique) et l'autre formé des couleurs jaune et même bleue. Les bonnes relations sont plus nombreuses que les mauvaises. La moyenne arithmétique est de $74,41 \%$, soit trois réponses identiques sur quatre dans les cartes. L'homogénéité est donc assez grande. En Basse-Navarre orientale et en Soule, la distance linguistique est élevée. Tous comptes faits, Urrugne indique donc que ce parler a dans l'ensemble d'assez bonnes relations avec les autres parlers, 70 parlers étant au-dessus de la moyenne arithmétique et 67 étant au-dessous.

Dans la carte 6, le point de référence est Bidarray (1321), en BasseNavarre occidentale. Cette carte redit en quelque sorte ce que nous a appris la carte précédente. L'affinité linguistique avec le Labourd et la BasseNavarre occidentale est bonne, soit avec 75 parlers. Cependant, on trouve dans cet espace des parlers qui ont avec Bidarray de mauvaises relations comme Hendaye (cela ne surprend pas selon nos données), mais aussi Biriatou (1205), Sare (1205), Larresore (1216), Les Aldudes (1317) et Ascarat (1319). Comme dans la carte précédente, l'affinité est réduite avec la Basse-Navarre orientale et la Soule. La même courbe assez équilibrée caractérise les cartes 5 et 6 .

La carte 7 sert en quelque sorte de repoussoir, basée sur le point de référence Pagolle. La carte montre des relations très mauvaises entre Pagolle et les communes voisines, et de bonnes relations ci et là en Basse-Navarre et Labourd. Si la méfiance introduite par le savoir philologique ne l'avait déjà fait, cette carte dénonce le texte de Pagolle comme non représentatif du parler de la commune. 
La carte 8 examine le profil de Mauléon (1249). Elle montre clairement la particularité de l'espace souletin, très cohérent, auquel s'ajoutent deux communes du pays de Mixe (Arberats 1273 et Camou-Sussaute 1282). D'ailleurs les autres parlers de Mixe montrent des relations avec Mauléon qui sont au-dessus de la moyenne arithmétique. Les relations avec les parlers labourdins ne sont pas extrêmement mauvaises, mais au total Mauléon présente davantage de mauvaises relations (80) que de bonnes (57).

\subsubsection{Au-delà des cartes de similarité : à la découverte d'aires}

La notion de noyau dialectal est étroitement liée à celle de dialecte. En effet, la concomitance territoriale de diverses caractéristiques linguistiques produit une homogénéité géotypologique. Le noyau dialectal fournit une ossature au dialecte et en réunit les caractéristiques linguistiques propres les plus prégnantes. Selon Hans Goebl (2003 : 80), le noyau dialectal doit remplir deux exigences :

- un tissu dialectal qui possède plus d'un noyau dialectal;

- un filet d'isoglosses entre les divers noyaux dialectaux qui constitue un amphizone ou zone de transition dans laquelle les traits dialectaux se mélangent tant et si bien que peu à peu ils laissent apparaitre un autre noyau lexical.

Pour repérer ces noyaux dialectaux, en tenant compte des distributions maximales que nous offrent les outils de statistique descriptive, on choisit le plus grand coefficient de similarité entre leurs relations. On obtient donc les parlers qui ont entre eux les coefficients maximaux de similarité. On choisit à cet effet l'indice $\operatorname{GIW}(1)_{\mathrm{jk}}$ qui renforce les caractéristiques à aire exiguë, au détriment des phénomènes à aire plus vaste. L'indice RIW $_{\mathrm{jk}}$ aurait permis de traiter toutes les caractéristiques sur un pied d'égalité. Cet indice pondéré est adéquat, car il souligne les caractéristiques isolées ou rares qui apparaissent dans les noyaux dialectaux. En effet, chaque noyau dialectal a besoin de ses caractéristiques propres et ce sont ces caractéristiques que l'indice marque davantage.

La carte 9 rend visibles les résultats d'une telle procédure. C'est une première approche de la structuration géolinguistique dans les parlers basques de Soule, Basse-Navarre et Labourd : les parlers sous couleurs rouge et orange sont ceux qui ont le plus haut indice de similarité avec leurs voisins. Les parlers sous couleur bleue sont ceux ayant le plus bas indice de similarité. Autrement dit, les parlers sous couleurs bleue et orange sont ceux qui offrent un espace linguistique plus homogène. Ces observations étant faites, les apports les plus significatifs de cette carte sont les suivants : 
a) en dehors de l'espace significatif qui apparaît à droite sur la carte, il n'y a pas de noyau dialectal très net dans les trois provinces selon les données obtenues dans le recueil Bourciez;

b) la plupart des parlers qui font partie des groupes situés au-dessus de la moyenne arithmétique sont pour la plupart en Soule. Ceci confirme que la Soule possède le noyau dialectal le plus net;

c) au milieu de la carte, la Basse-Navarre offre un ensemble de parlers aux nombreuses failles et ruptures (la couleur bleue y est très présente);

d) au Labourd, on peut deviner des embryons de noyau dialectal (à Saint-Pée-sur-Nivelle par exemple);

e) c'est en Basse-Navarre que se trouvent la plupart des parlers qui offrent les caractéristiques les plus étendues, mais aussi les 9 parlers qui présentent les traits les plus originaux. La plupart de ces parlers sont en Basse-Navarre orientale : Saint-Jean-Pied-de-Port (1326), Aincille (1329), Hosta (1305), Bunus (1303), Arhansus (1301), UhartCize (1298), Arbérats (1273), Arbouet-Sussaute (1275) et CamouSuhast (1282).

On ne peut donc parler que d'un seul noyau dialectal, situé en Soule, les autres régions n'ayant pas d'autres noyaux sinon comme embryons peu solides.

\subsection{Présentation de la carte 10 : carte des écarts-types}

L'écart-type est un outil classique de la statistique descriptive (Goebl, 2002 : 24). La synopse des écarts-types sert surtout à repérer les parlerspont ou les zones de transition. À définir ce qu'est une zone de transition ou un parler-pont, nous dirions qu'il s'agit des parlers intermédiaires situés entre les noyaux dialectaux signalés ci-dessus. La caractéristique de ces parlers est de posséder peu de caractères propres et d'offrir peu à peu le «chemin» ou «pont» qui permet de passer d'un système linguistique à un autre. L'écart-type permet d'isoler et de mettre en évidence ces parlers situés entre noyaux dialectaux à forte cohésion.

Dans la carte 10, deux noyaux dialectaux se dessinent de part et d'autre de l'espace considéré, en Labourd et en Soule. Entre ces deux noyaus assez nets, on trouve les parlers de transition (colorés en bleu ou jaune). L'ensemble des parlers bas-navarrais serait concerné et constituerait une vaste zone de transition. On notera que cette zone connaît tout de même une rupture plus forte avec la Soule qu'avec le Labourd. Cette structuration de l'espace linguistique révélée par cette carte ne manque pas d'importance 
puisqu'il s'agit de la synthèse des données de 151 cartes lexicales de 137 parlers.

\subsection{Synopse des coefficients d'asymétrie de Fisher (carte 11)}

Nous n'avons pas insisté jusqu'ici sur le graphique de distribution des fréquences qui apparaît en bas à droite de nos cartes. Un simple regard permet de voir que cette distribution varie d'une carte à l'autre. Le fait que cette courbe soit symétrique ou asymétrique est une donnée d'importance. L'un des outils les plus connus pour mesurer ceci est appelé coefficient d'asymétrie de Fisher. On dit que la distribution des fréquences est symétrique quand la distribution des fréquences est également répartie de part et d'autre de la moyenne arithmétique. Au contraire, l'asymétrie apparaît si l'un des côtés est nettement plus important, L'asymétrie apparaîtra à droite (valeurs positives) quand le nombre de fréquences au-dessus de la moyenne arithmétique est plus élevé et l'asymétrie apparaîtra à gauche (valeurs négatives) quand le nombre de fréquences au-dessous de la moyenne arithmétique est plus élevé. Le coefficient est 0 quand la courbe est symétrique. On considére généralement que, d'un point de vue géolinguistique, les parlers qui ont obtenu des fréquences supérieures à la moyenne arithmétique (valeurs positives) sont des parlers conservateurs, tandis que les parlers qui ont obtenu des valeurs négatives ont de bonnes compétences communicatives avec les autres parlers.

Dans la carte 11, deux aires conservatrices apparaissent, en Labourd et Soule, de couleur rouge, aux deux extrémités de la carte. D'un point de vue géolinguistique, ces aires ont de mauvaises relations linguistiques avec les parlers voisins et conservent bon nombre de caractéristiques propres. Inversement, en jaune et bleu, les parlers aux valeurs négatives ont de bonnes relations avec les autres parlers et en même temps peu de caractéristiques propres. Apparaît ici un point de vue antagoniste à celui dégagé par la carte 10 : alors que la carte 10 montrait des parlers de transition, la carte 11 montre surtout des parlers qui ont peu évolué.

\section{Dialectométrie multidimensionnelle ou dendographique}

La dialectométrie multidimensionnelle permet de franchir un pas supplémentaire dans l'étude de la structuration géolinguistique. Elle s'appuie sur la classification ascendante hiérarchique $(\mathrm{CAH})$. Parmi les outils que nous avons choisi pour la classification automatique, nous utilisons les dendogrammes qui sont représentés sous forme d'un arbre logique. Dans cette classification, tous les parlers sont pris en compte. Sur la carte 13 tous ces 
dendrogrammes sont placés sur la gauche au début de la ligne. Ces parlers sont situés en fonction de leur proximité linguistique. Chaque groupe rassemble l'ensemble des parlers qui en font partie. Cette procédure de classification est fréquemment utilisée en sciences sociales (sociologie...), en linguistique (glottochronologie), dont la dialectologie et la géolinguistique : Goebl (1991, 1992), Aurrekoetxea (1995), Bisquerra (1989), Jambu (1989).

\subsection{Explication du dendrogramme de la carte 12}

Prenons l'image de l'arbre représenté sur le dendrogramme comme s'il était à l'envers, sa cime vers le bas. Les barres horizontales qui partent de la droite de l'image en sont les branches maîtresses. De ces branches surgissent d'autres branches plus réduites. Chaque parler est représenté par une branche, soit 137 branches en tout.

Cet outil classificatoire distribue les branches (les parlers) en fonction de leur ressemblance. Les plus proches sont ceux qui se ressemblent le plus. Si deux parlers avaient absolument toutes les caractéristiques communes ils seraient représentés au niveau 0 du dendrogramme. Plus l'intersection des branches (des parlers) est située à droite et moins les parlers en questions ont de ressemblance. À la fin de la procédure, tous les parlers sont réunis dans un groupe, ce qui n'a pas d'intérêt géolinguistique. Plus l'intersection a lieu à gauche et plus la cohésion des parlers concernés est grande. La carte 12 montre les résultats de cette procédure. Les premières étapes apparaissent sur cet arbre hiérarchique. Dans ce dendrogramme sont de couleur rouge les 7 premiers parlers à un niveau très bas. Les deux parlers ayant le plus de ressemblance sont Camou-Suhast et Arbérats-Sillègue (1282 et 1273) en Mixe; en Soule on trouve Lichans-Sunhar (1354) et Etchebar (1349), le troisième groupe étant formé par Hosta (1305) et Bunus (1303). Le premier parler qui s'est regroupé avec Camou-Suhast et Arbérats-Sillègue est le parler de Arbouet-Sussaute (1275).

\subsection{Présentation des cartes 13 et 14}

La carte 14 présente la carte et le dendrogramme. La carte n'est rien d'autre que la projection géolinguistique du dendrogramme. Ici encore, on procède à un regroupement des parlers en quatre groupes aux couleurs distinctes. La couleur de chaque groupe est celle portée sur la carte. Sur la carte, quatre groupes cohérents apparaissent, sauf une exception :

a) le groupe de couleur rouge regroupe la plupart des parlers du Labourd et quelques-uns de Basse-Navarre. L'aire est assez cohérente, 
même si on y trouve Larribar (1290) et Luxe-Sumberraute (1292). Et cette aire comprend en vert des parlers qui sont regroupés dans le groupe suivant : il s'agit de Ciboure (1206), Mouguerre (1194) et Jatxou (1215);

b) le groupe de couleur verte regroupe les parlers de Basse-Navarre occidentale. Il constitue une aire dense et cohérente (si on élimine les trois communes citées ci-dessus). Un autre groupe discontinu au nord est formé par Urcuit (1196), Bardos (1194) et Isturitz (1238). On trouve deux autres communes isolées en Mixe : Amendeuix (1271) et Garris (1286). Enfin, Saint-Just-Ibarre (1313) et Pagolle (1297) forment une autre micro-aire;

c) le groupe de couleur bleu clair regroupe les parlers de Basse-Navarre orientale et Domezain-Berraute (1283) de Soule. 23 parlers sont ainsi regroupés, essentiellement situés en pays de Mixe, plus Lantabat, Domezain et 7 communes du pays d'Ostabarret. Cette aire est très cohérente et n'a pas de parler hors de son aire continue, même si elle enclave deux communes regroupées ici dans le premier groupe (rouge);

d) le groupe de couleur bleu sombre regroupe tous les parlers souletins, sauf Domezain qui «marche» avec le pays de Mixe, et Pagolle dont nous avons déjà signalé le peu de pertinence. La cohérence de ce groupe est étonnante et très supérieure à celle des trois autres.

Le dendrogramme présente donc ici quatre groupes et c'est le choix du chercheur, car c'est un niveau d'analyse qui montre une répartition géolinguistique assez proche de la fameuse carte des dialectes basque élaborée par Louis-Lucien Bonaparte à une date à peine plus ancienne que les données du recueil Bourciez que nous utilisons ici. Le chercheur peut donc jouer sur le nombre de regroupements dans le dendrogramme selon les phénomènes qu'il veut étudier.

La carte 14 ne présente que quelques différences: elle utilise l'algorithme IPI(1) $)_{\mathrm{jk}}$ qui pondère les données. L'aire rouge et l'aire verte sont les plus concernées, très peu l'aire bleu clair et pas du tout l'aire bleu foncé. Si nous poussons le dendrogramme vers trois groupes seulement, l'aire souletine en bleu foncé continue à rester la même. Par contre, il y a un regroupement de la Basse-Navarre occidentale et du Labourd qui forme une aire rouge plus vaste, la Basse-Navarre orientale continuant à constituer une aire à part, de dimension plus réduite. 


\section{Une première comparaison entre les cartes obtenues par procédure classificatoires et la carte de Louis-Lucien Bonaparte}

Nous pouvons esquisser une première ébauche de comparaison entre les cartes produites ici et leurs résultats et la fameuse carte des dialectes basques proposée à Londres par Louis-Lucien Bonaparte au XIX ${ }^{\mathrm{e}}$ siècle.

a) d'une part, nous faisons mine d'ignorer ici toutes les discussions sur la nature des dialectes, sur leur existence même;

b) d'autre part, la démarche de L.-L. Bonaparte est totalement différente de celle suivie ici. Quant aux données elles-mêmes, les nôtres ne reposent pour l'instant ici que sur des faits lexicaux alors qu'on sait que Bonaparte privilégia les données d'ordre morphologique. Travaillant «à la main», sans concours d'aucun outil mathématique ou de classification, et pour cause, Bonaparte avait sélectionné un nombre d'objets linguistiques assez réduits à partir desquels il distinguait les dialectes et sous-dialectes ;

c) les procédures classificatoires ne laissent préjuger de rien quant à la structuration géolinguistique. Nous avons ici fourni les données de 151 cartes et 151 parlers sans chercher à l'avance qu'elles nous indiquent une distribution particulière dans l'espace;

d) nous avons cependant utilisé deux indices de similarité qui fournissent une structuration légèrement différente, l'indice pondéré contribuant à fournir une carte dans laquelle les faits linguistiques minoritaires sont surreprésentés, ce que l'autre indice de similarité ne fait pas.

On sait comment Bonaparte en vint peu à peu à diviser les parlers des trois provinces de Soule, Basse-Navarre et Labourd en quatre dialectes : labourdin, bas-navarrais occidental, bas-navarrais oriental et souletin. Pour le dialecte labourdin, il proposait trois sous-dialectes : le labourdin propre (avec trois variantes, Saint-Jean-de-Luz, Sare et Ainhoa), et le labourdin hybride (Arcangues). Pour le bas-navarrais occidental et oriental il proposait trois sous-groupes. Pour le souletin, il proposait deux sous-dialectes : le souletin proprement dit et le roncalais (celui-ci en dehors ici de notre champ d'études puisque situé en Navarre).

Une comparaison entre la fragmentation dialectale selon Bonaparte et la nôtre en carte 14 montre quelques changements, qui ne sont pas cependant d'une ampleur considérable.

S'agissant du labourdin, les communes qui limitent ce dialecte sont Lahonce (1193), Mouguerre (1194), Jatxou (1215), Cambo (1222) et Itxassou 
(1223). À l'intérieur du domaine labourdin, deux communes semblent montrer une plus grande proximité avec le bas-navarrais occidental : Ciboure (1206) et Villefranque (1218).

Alors que Bonaparte classe Lahonce et Mouguerre comme bas-navarraises, notre carte les signale comme labourdines. De même, Bonaparte classe la variété d'Ustaritz comme du bas-navarrais occidental alors que notre carte la donne comme labourdine. Concernant le bas-navarrais occidental, les communes limitrophes à l'ouest sont Urcuit (1196), Briscous (1238), Hasparren (1227), Macaye (1229), Louhossoa (1224), Bidarray (1231). À l'est les communes limitrophes sont Bardos (1243), Amorots (1272), Meharin (1230), Armendaritz (1302), Iholdy (1299), Suhescun (1314), Ainhice-Mongelos (1330), Lecumberry (1339) et Mendive (1340).

Ici, la différence entre la carte Bonaparte et la nôtre réside dans le fait que bon nombre de parlers que Bonaparte situait dans l'un ou l'autre basnavarrais apparaissent dans notre carte comme faisant partie du bas-navarrais occidental. Autrement dit, dans notre carte, le bas-navarrrais occidental couvre une aire plus étendue que dans la carte de Bonaparte.

Pour ce qui est du bas-navarrais oriental, cette aire recouvre les parlers du pays de Mixe et les communes de Lantabat (1309), Larceveau (1310), Ibarrole (1306) et Hosta (1310). Hormis ces quatre parlers, la carte de Bonaparte et la carte 14 montrent la même aire quant au bas-navarrais oriental. Quant au souletin, à part le cas de Pagolle (non fiable dans nos données Bourciez) et celui de Domezain (1283), la carte de Bonaparte et la nôtre diffèrent sur le parler de Lohitzun. Bonaparte le donne comme basnavarrais oriental et nous comme souletin.

\section{Conclusion}

Il nous apparaît que ces trois ouvrages fournissent une information à prendre en compte pour la distribution géolinguistique des objets linguistiques repérés sur le territoire concerné en Soule, Basse-Navarre et Labourd. Nous avons conscience de ne pas avoir épuisé le corpus. Il mérite encore un approfondissement et l'ouverture de divers chantiers à venir est souhaitable.

Une étude pourrait mesurer l'influence des langues voisines. On peut y consacrer des travaux précis sur une micro-aire, par exemple une expertise pour savoir si les pays de Mixe ou d'Ostabarret sont, comme on le dit souvent, plus ouverts à l'influence romane, occitane surtout, que les espaces voisins. Il faut pour cela choisir une palette de cartes fournissant une telle information. Du point de vue diachronique, la photographie des parlers basques donnée par Bourciez en 1895 peut fournir un point de départ pour 
une comparaison avec le basque parlé plus d'un siècle plus tard sur les mêmes espaces. Qu'est-ce qui a changé ? Jusqu'à quel point? Qu'est-ce qui demeure fondamentalement identique? Autant de questions dans un contexte qui montre des parlers en perdition face au français ou en évolution depuis l'apparition de standards en basque.

On peut procéder à une interprétation plus fine de ces données que dans nos cartes qui ne fournissent qu'un point de vue général. Nous avons constaté que deux articles ont ouvert cette voie, l'un sur les apports lexicaux du recueil (Ormaetxea, 2005), l'autre sur un point de déclinaison (Iglesias, 2005). Une étude serait à faire sur la manière dont un texte d'abord oral, fourni de vive voix par des locuteurs locaux, a été retranscrit par les instituteurs. Certes, bien des marques de cette oralité subsistent dans ces documents. Mais les collecteurs n'ont pas opéré les mêmes choix. L'orthographe des textes fournit une mine de renseignements à exploiter. C'est justement cette même orthographe que les instituteurs citent très souvent comme une des difficultés les plus prégnantes à l'heure de traduire, comme si les autres difficultés attendues, telles que la syntaxe, leur semblaient peu marquées. Ce travail sur l'orthographe, qui prolongerait la belle thèse de J. A. Mujika (2002), reste à faire. Une étude pourrait permettre de comparer ces versions avec celles données en d'autres langues. On sait que la Parabole de l'Enfant prodigue, revue et corrigée, a constitué un texte étalon traduit en de nombreuses langues; on songe aux travaux des Coquebert de Montbret par exemple (Levasseur, 1985; Oyharçabal, 1992; Simoni-Aurembou, 1989). Faut-il signaler que nous attendons avec impatience la parution des documents réunis par Bourciez en Gascogne même où ce sont des centaines de versions qui dorment et méritent mise à disposition du public? La comparaison avec les phénomènes observés dans d'autres langues permettrait de relativiser nos propres analyses.

Enfin, au risque de nous éloigner de la dialectologie, une étude sur les instituteurs qui ont écrit ces documents serait d'un grand intérêt pour l'histoire sociale de la langue. D'une part, on peut repérer ceux plus à l'aise en basque que d'autres, certainement béarnais. Mais une étude sur archives dans les dossiers d'instituteurs permettrait d'affiner l'image des hussards de la République dont on se contente, bien rapidement. De manière anecdotique, un simple sondage nous a permis ainsi de relever comment un inspecteur venant visiter un de ces instituteurs est vivement pris à partie par l'épouse de l'instituteur. L'inspecteur ne doute pas que les paroles qui lui sont adressées lui soient peu cordiales, mais il ne peut en donner la teneur exacte car cette épouse parle... en basque, la langue basque semblant être la langue quotidienne du couple hors de l'école. Ces instituteurs formaient un réseau, à partir de la fraternité acquise en école normale. Ils sont aussi 
connus parfois pour leur collaboration avec les collecteurs de textes basques (chansons, contes, traductions) tels que Cerquand ou Azkue. C'est le cas de l'instituteur Constantin par exemple. À Ostabat, les deux versions sont fournies par deux rivaux, l'une par Primorena, qui fut collaborateur de Azkue, l'autre par Adolphe Jaureguy, père de deux célèbres joueurs de rugby, l'un, Adolphe, capitaine de l'équipe de France à maintes reprises. Enfin, on y trouvera des personnages moins connus comme Chimits (parent de Jean Haritschelhar), qui fait part de ses réflexions personnelles sur l'orthographe à adopter en langue basque.

\section{RÉFÉRENCES BIBLIOGRAPHIQUES}

AlLières Jacques, 1978, «Petit atlas linguistique basque français, Bourciez 1 » (20 cartes commentées), Fontes Linguae Vasconum, n ${ }^{\circ}$ 27, p. 353-386.

AurRéoetXeA Gotzon, 1995, Bizkaieraren egituraketa geolinguistikoa, Leioa, UPV-EHUko Argitarapen Zerbitzua / Bilbao, Université du Pays basque.

AurReKOetXeA Gotzon, 2004, «Iparraldeko hizkeren sailkapena (1): lexikoa», $A S J U$, vol. $38, \mathrm{n}^{\circ} 1, \mathrm{p} .287-331$.

AurRekoetXea Gotzon \& Videgain Charles (éds), 2004, Haur prodigoaren parabola Ipar Euskal Herriko 150 bersiotan [La parabole de l'Enfant prodigue en 150 versions basques], Anuario del Seminario de Filología Vasca Julio de Urquijo, annexe ${ }^{\circ}$ XLIX, Bilbao, Service de publications de l'Université du Pays basque.

AurrekoetXea Gotzon, Iglesias Aitor \& Videgain Charles, 2007 [2004], «Bourciez Bildumako Euskal Atlasa (BBEA-1): 1. Lexikoa [El atlas lingüístico Bourciez: 1. Léxiko]», ASJU, vol. $38, \mathrm{n}^{\circ} 2$.

AurrekoetXea Gotzon, Iglesias Aitor \& Videgain Charles, 2008 [2005], «Bourciez Bildumako Euskal Atlasa (BBEA-2): 2. Gramatika [El atlas lingüístico Bourciez: 2. Gramática]», ASJU, vol. 39, $\mathrm{n}^{\circ} 1$.

Azkue Resurrección María de, 1922, Cancionero popular vasco, 2 vol., Barcelone, Boileau \& Bernusconi.

Balley Kenneth D., 1994, Typologies and Taxonomies: An Introduction to Classification Techniques, Iowa City, Sage Publications.

BISQUERRA Rafael, 1989, Métodos de investigación educativa, Barcelone, CEAC.

BouRCIEZ Édouard, 1895, «Introduction au Recueil des idiomes de la région Gasconne», Revue des Pyrénées et de la France méridionale, p. 321-324.

Chandon Jean-Louis \& Pinson Suzanne, 1981, L'analyse typologique : théorie et applications, Paris, Masson.

Goebl Hans, 1981, «Éléments d'analyse dialectométrique (avec application à l'AIS)», RLiR, no 45 , p. 349-420.

Goebl Hans, 1987, «Points chauds de l'analyse dialectométrique : pondération et visualisation », RLiR, $\mathrm{n}^{\circ} 51$, p. 63-118. 
Goebl Hans, 1991, «Una classificazione gerarchica di dati geolinguistici tratti dall'AIS. Saggio di dialettometria dendrografica », Linguistica, no 31 , p. 341351.

Goebl Hans, 1992, «Problèmes et méthodes de la dialectométrie actuelle (avec application à l'AIS)», dans Actes du Congrès international de dialectologie (Bilbao, 1991), Bilbao, Euskaltzaindia, p. 429-475.

Goebl Hans, 2002, «Analyse dialectométrique des structures de profondeur de $l^{\prime} \mathrm{ALF} \gg, R L i R, \mathrm{n}^{\circ} 66$, p. 5-63.

Goebl Hans, 2003, «Regards dialectométriques sur les données de l'Atlas linguistique de la France ( $A L F)$ : relations quantitatives et structures de profondeur», Estudis Romànics, $\mathrm{n}^{\circ} 25$, p. 59-96 + 24 cartes.

Goebl Hans, 2005, «La dialectométrie corrélative : un nouvel outil pour l'étude de l'aménagement dialectal de l'espace par l'homme», RliR, nº 69, p. 321-367.

Goebl Hans, 2006, «Sur le changement macrolinguistique survenu entre 1300 et 1900 dans le domaine d'oïl. Une étude diachronique d'inspiration dialetométrique », Linguistica, $\mathrm{n}^{\circ}$ 46, p. 1-37.

Gordon Deborah, 1999, Ants at Work: How an Insect Society Is Organised, New York, The Free Press.

JAMBU Michel, 1989, Exploration informatique et statistique des données, Paris, Dunod.

Levasseur Florence, 1985, L'enquête linguistique de Coquebert de Montbret (1806-1812), mémoire de maîtrise de lettres (dirigé par J. B. Marcellesi), Université de Rouen.

MujIKA Jose Antonio, 2002, Euskal ortografiaren hastapenak Iparraldeko Literaturan [Les débuts de l'orthographe en basque dans la literature du Pays basque nord], thèse de doctorat, Université du Pays basque, Lejona.

OyharçABAl Beñat, 1992, «Euskararen mugak hego aldean 1807.ean: Eugène Coquebert de Montbret-en artxiboetan gelditu dokumentuak», dans Euskal Dialektologiako Kongresua. Anejos del Anuario del Seminario de Filología Vasca "Julio de Urquijo" (ASJU-n argitaratzekoa), nº 28, p. 241-253.

Simoni-Aurembou Marie-Rose, 1989, «La couverture géolinguistique de l'Empire français : l'enquête de la Parabole de l'Enfant prodigue», dans Espaces romans. Études de dialectologie et géolinguistique offertes à Gaston Tuaillon, Grenoble, ELLUG, vol. 2, p. 114-139.

Sneath Peter H. \& Sokal Robert R., 1973, Numerical Taxonomy: The Principles and Practice of Numerical Classification, San Francisco, W. H. Freeman.

VIAUT Alain, 1995, «Bourciez et les études gasconnes», dans L'ethnologie à Bordeaux. Hommage à Pierre Métais (Actes du colloque du 10 mars 1994, Bordeaux), Département d'anthropologie sociale-ethnologie, Centre d'études et de recherches ethnologiques, Université Bordeaux 2, p. 109-128.

VIDEGAIN Charles, 1989, Le vocabulaire de l'élevage en pays d'Oztibarre. Contribution aux archives de l'oralité basque, thèse de doctorat (dirigée par J. Haritschelhar \& J. L. Fossat), Université Bordeaux 3. 


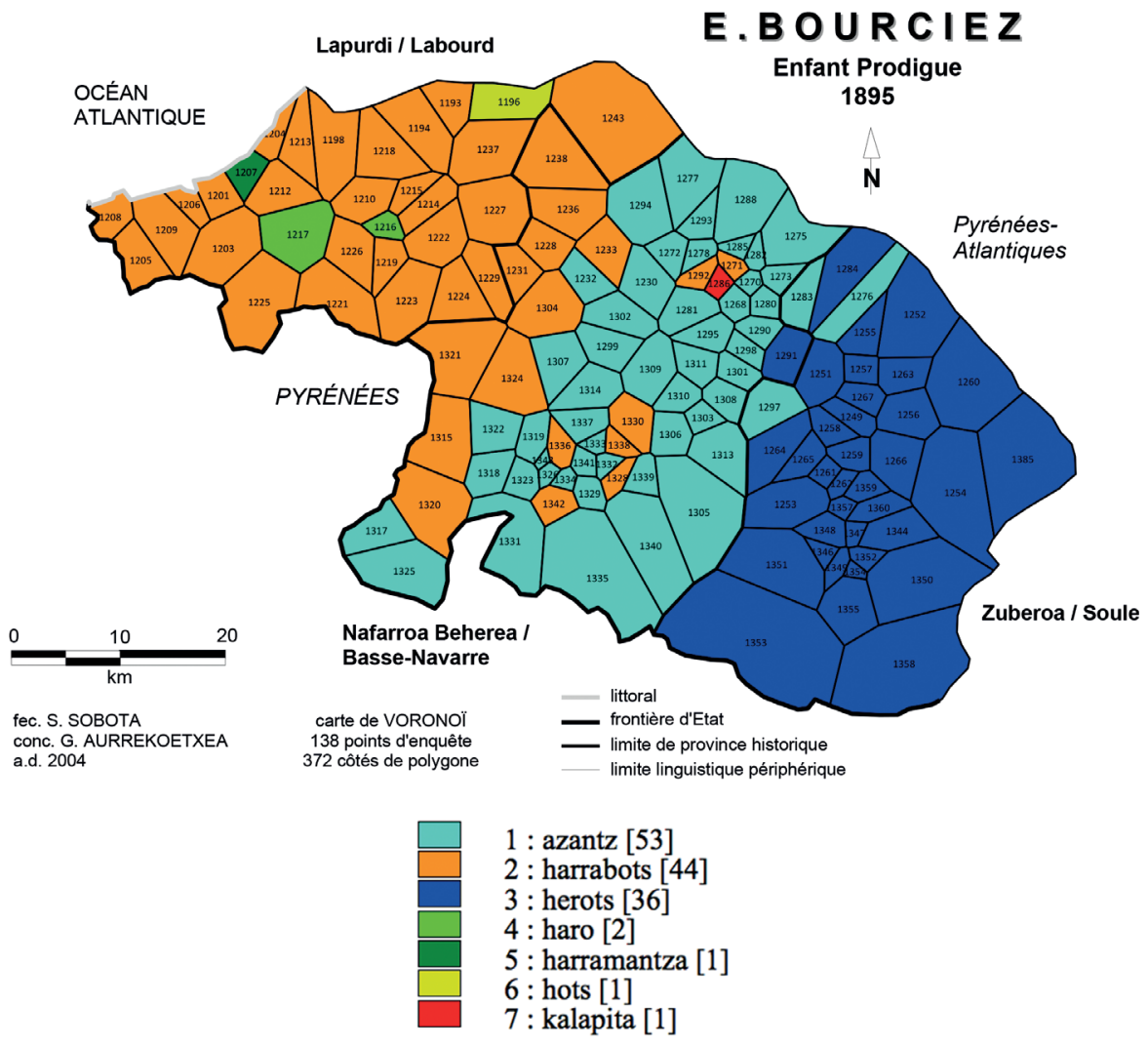

Carte 1. - Bruit. 


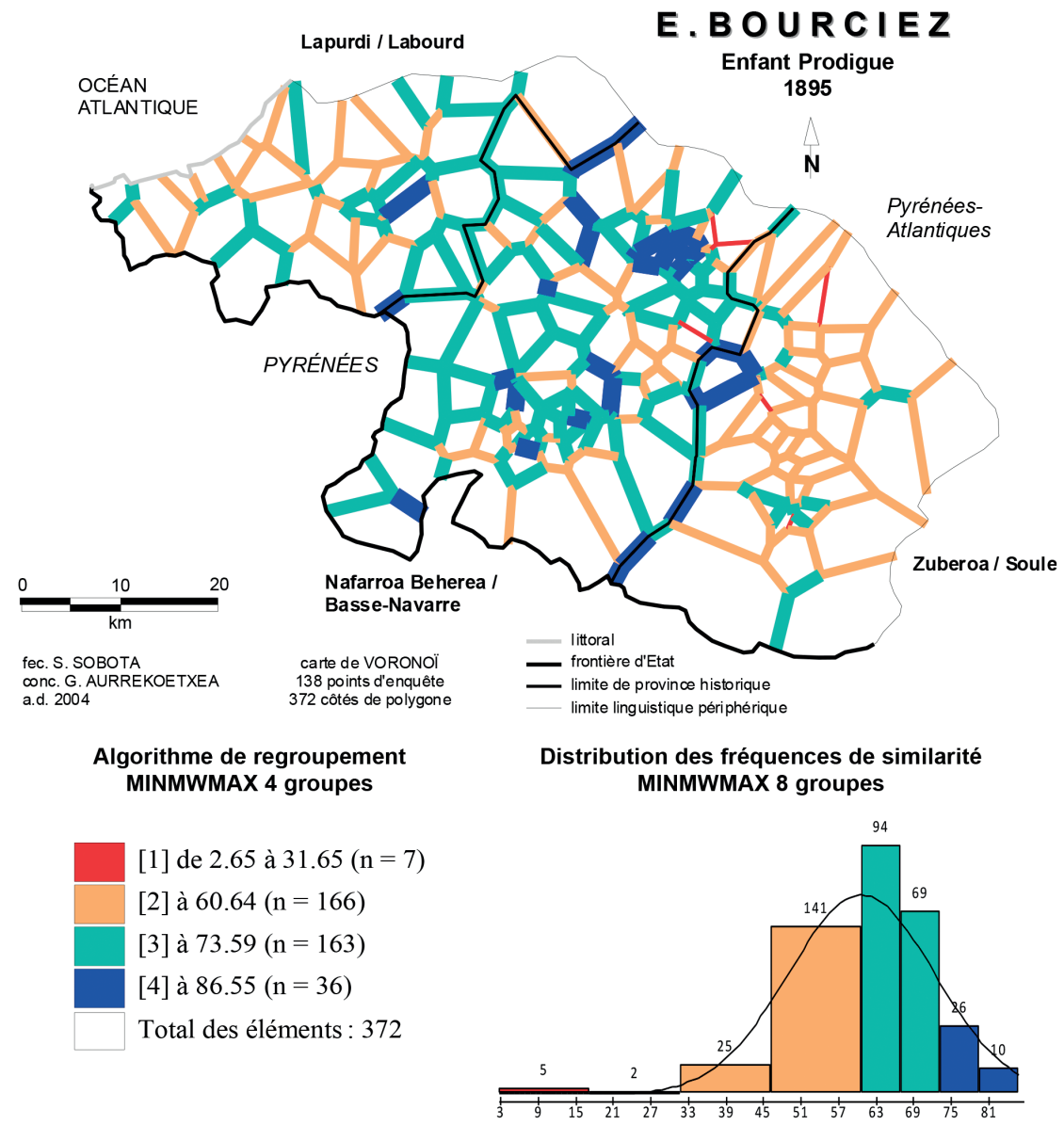

Carte 2. - Carte interponctuelle montrant la fonction distinctive des parlers (isoglosses synthétiques et quantitatifs). Corpus : 151 cartes lexicales. Indice de distance IPD(1) ${ }_{j k}$ ou GIW. Algorithme de regroupement MINMWMAX 4 groupes. 


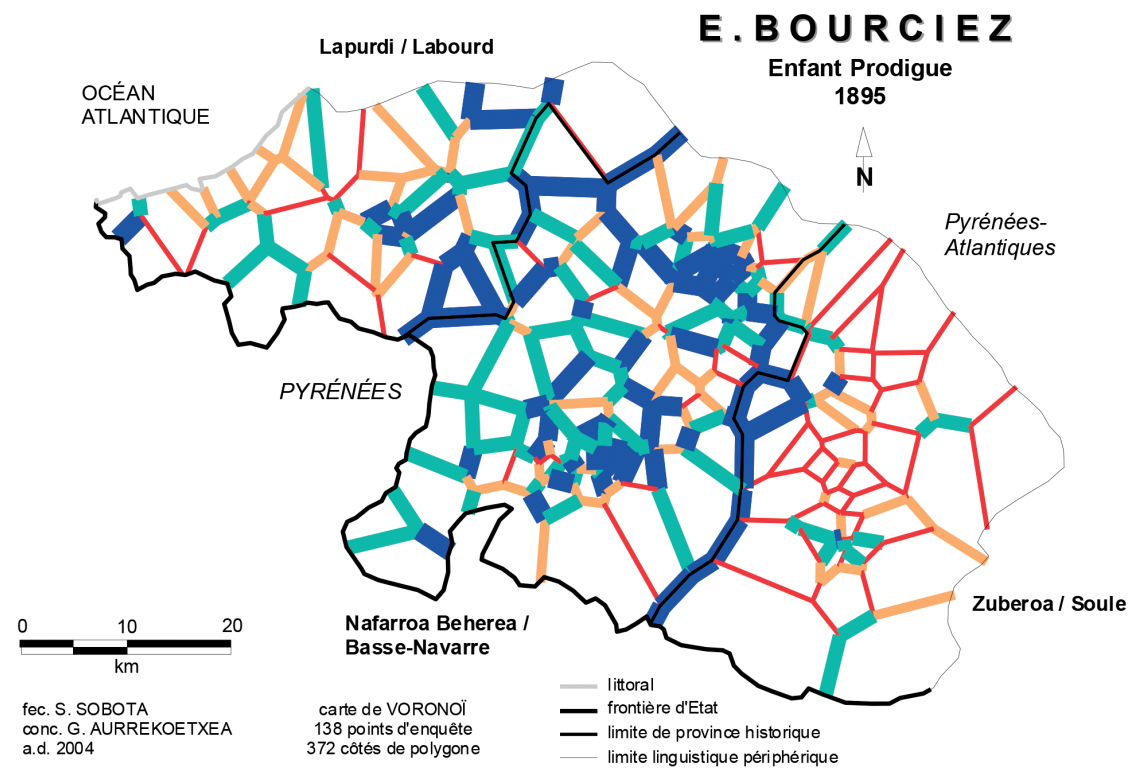

Algorithme de regroupement MedMw 4 groupes

Distribution des fréquences de similarité MedMw 8 groupes

[1] de 2.65 à $54.59(\mathrm{n}=87)$
$[2]$ à $60.63(\mathrm{n}=86)$
$[3]$ à $67.97(\mathrm{n}=100)$
$[4]$ à $86.55(\mathrm{n}=99)$
Total des éléments : 372

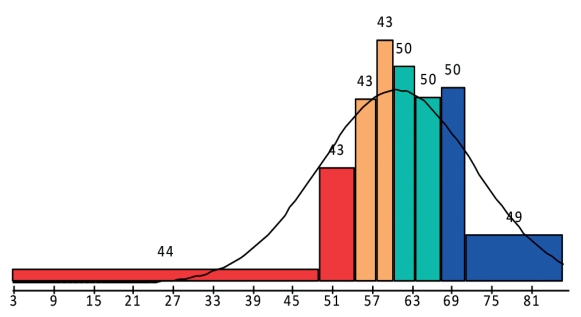

Carte 3. - Carte interponctuelle montrant la fonction distinctive des parlers (isoglosses synthétiques et quantitatifs). Corpus : 151 cartes lexicales. Indice de distance IPD $(1)_{j k}$ ou GIW. Algorithme de regroupement MedMw 8 groupes. 


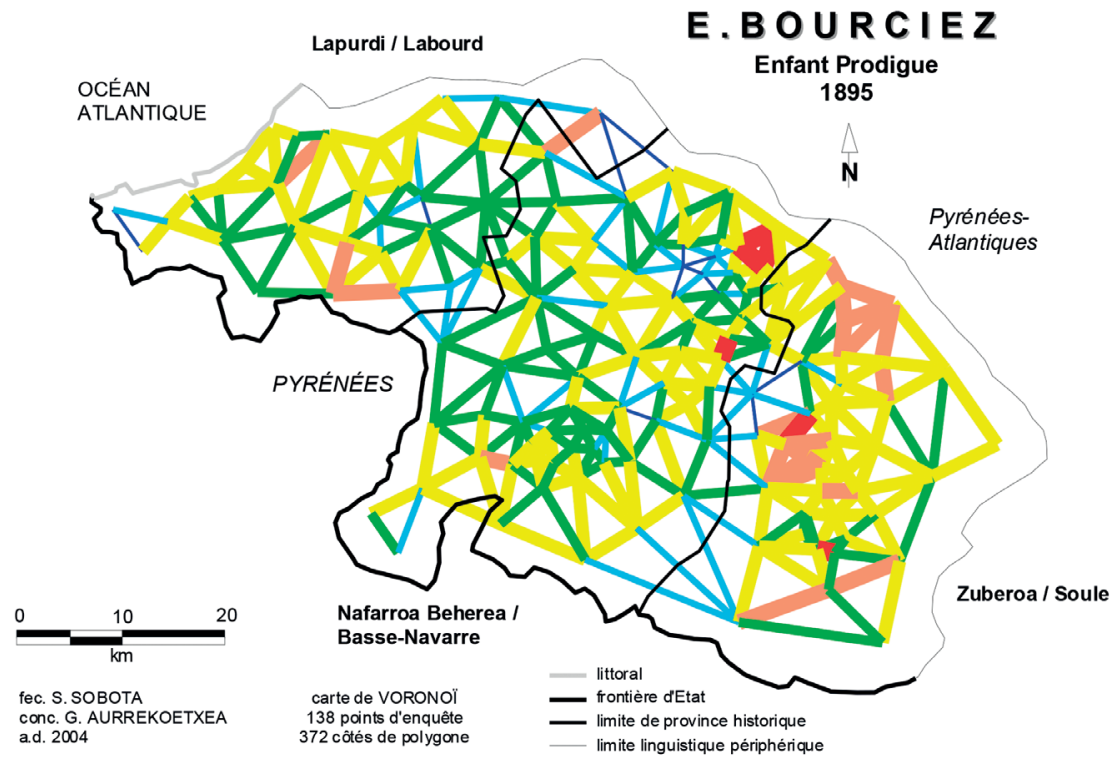

Algorithme de regroupement MINMWMAX 6 groupes

[1] de 64.79 à $69.55(\mathrm{n}=12)$

[2] à $74.32(\mathrm{n}=48)$

[3] à $79.08(\mathrm{n}=121)$

[4] à $85.83(\mathrm{n}=162)$

[5] à $92.58(\mathrm{n}=23)$

[6] à $99.33(\mathrm{n}=6)$

Total des éléments : 372
Distribution de la fréquence de similarité MINMWMAX 12 groupes

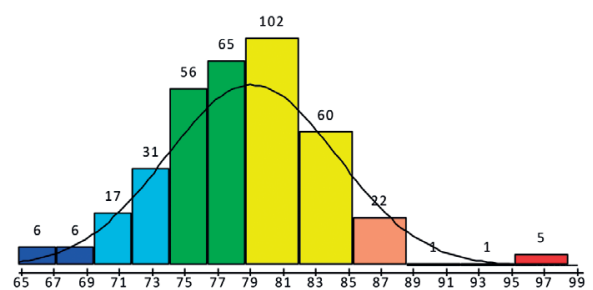

Carte 4. - Carte ponctuelle montrant la fonction communicative entre les parlers. Corpus : 151 cartes lexicales. Indice de distance RIW. Algorithme de regroupement MINMWMAX. Carte réalisée avec la triangulation de Delaunay. 


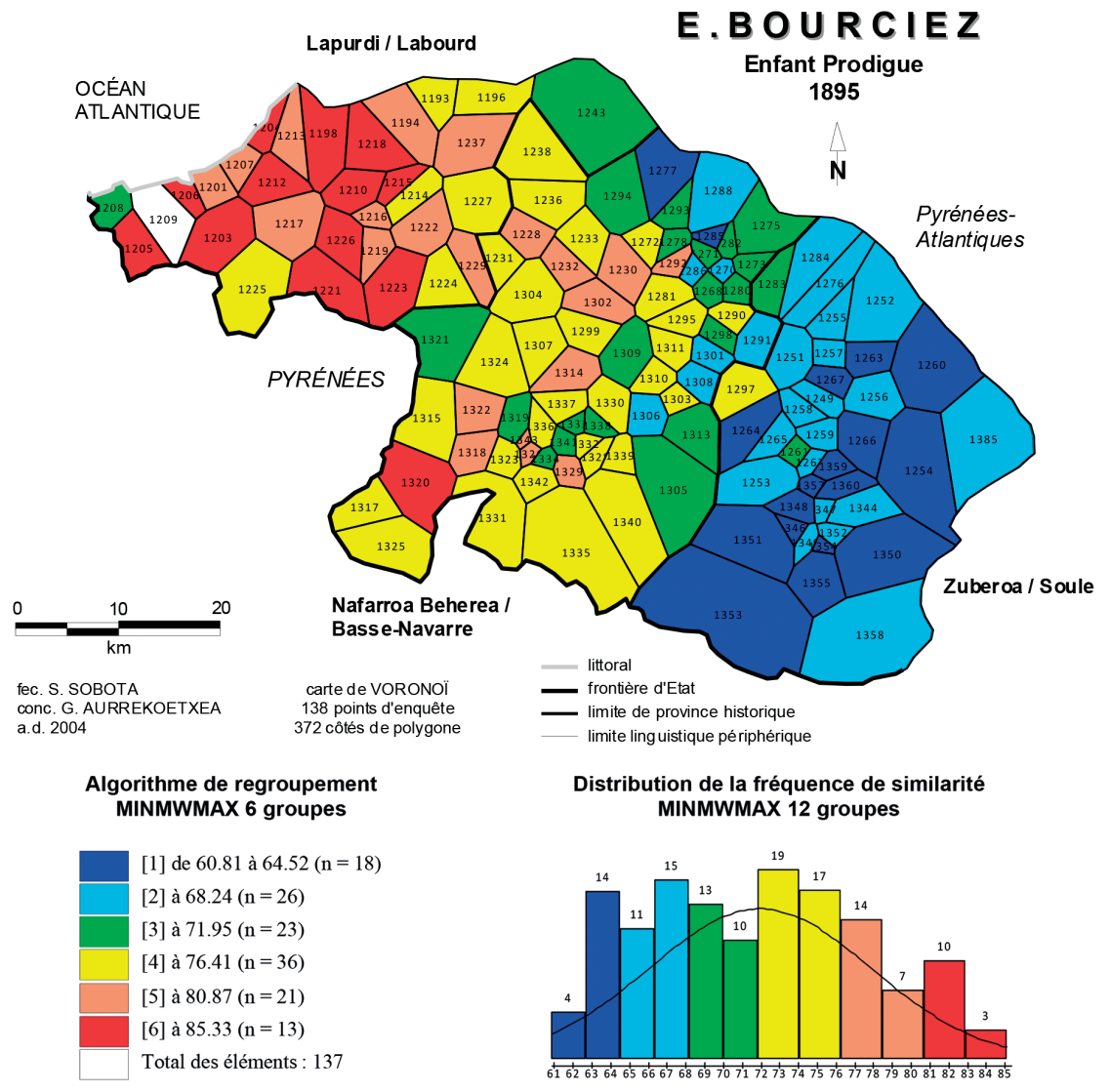

Carte 5. - Carte choroplèthe de similarité $\left(R / W_{1209, k}\right)$ appliquée au point de référence Urrugne (1209). Corpus : 151 cartes lexicales. Indice de distance RIW $W_{j k}$. Algorithme de regroupement MINMWMAX. 


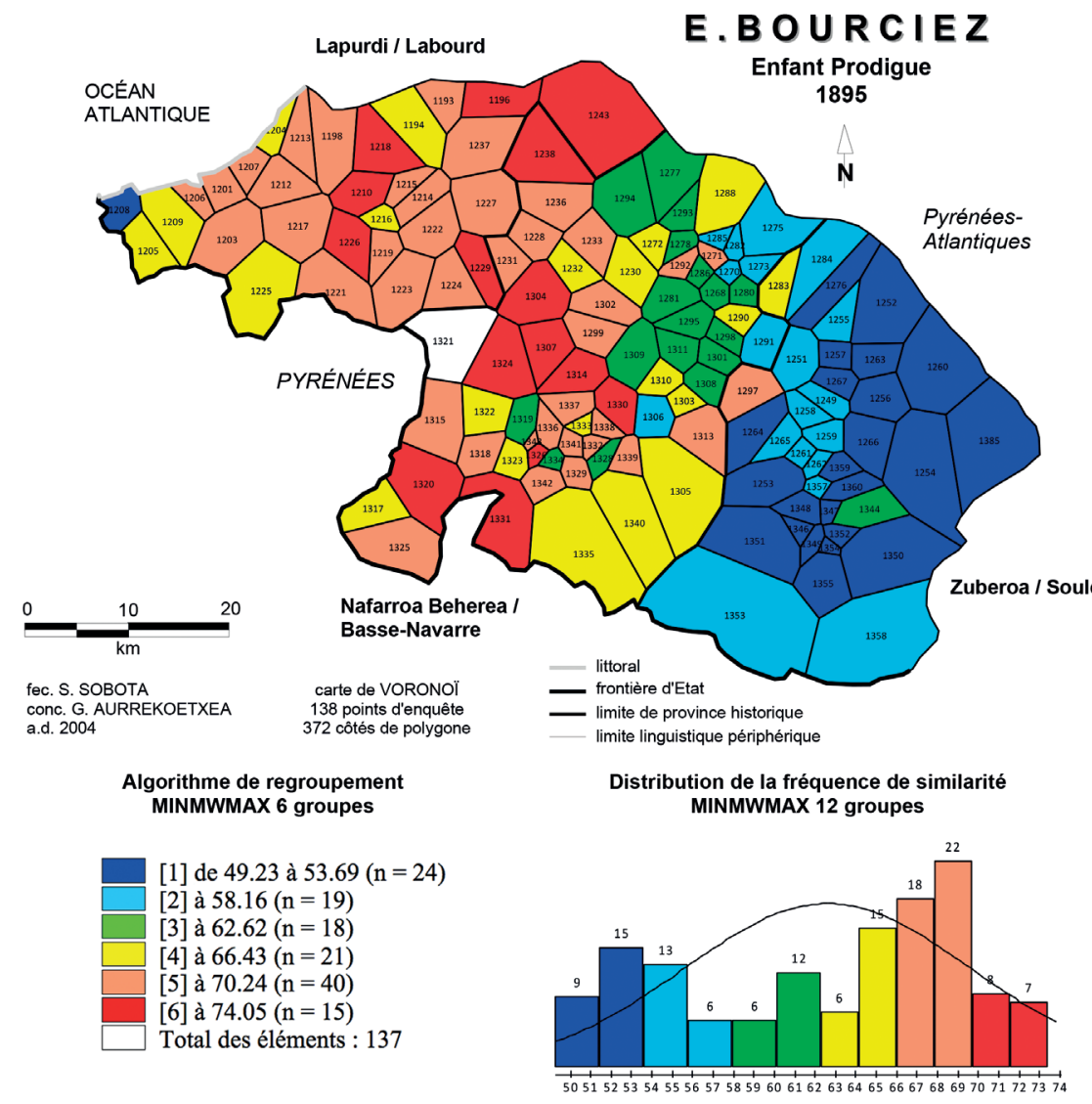

Carte 6. - Carte choroplèthe de similarité $\left(R / W_{1209, k}\right)$ appliquée au point de référence Bidarray (1321). Corpus : 151 cartes lexicales. Indice de distance $R I W_{j k}$. Algorithme de regroupement MINMWMAX. 


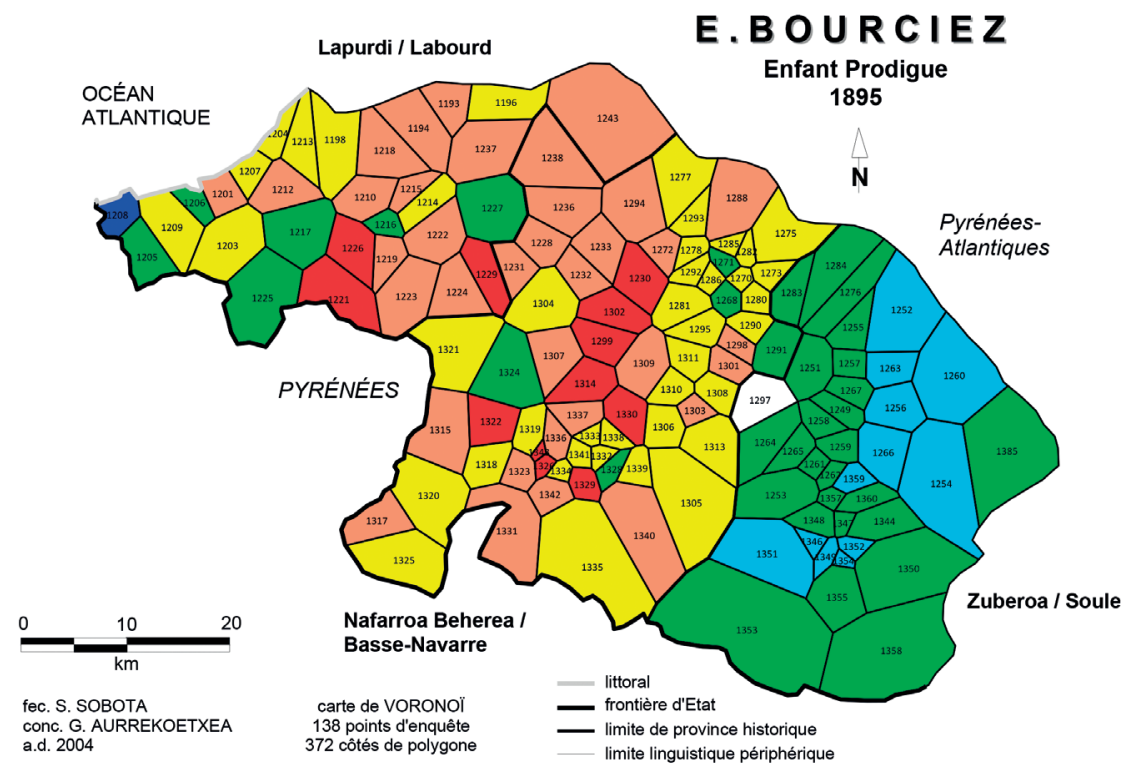

Algorithme de regroupement MINMWMAX 6 groupes

Distribution de la fréquence de similarité MINMWMAX 12 groupes
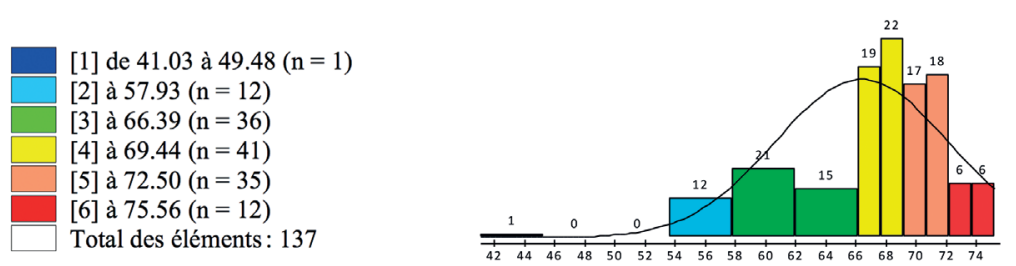

Carte 7. - Carte choroplèthe des distributions d'indice de similarité $\left(R / W_{1297, k}\right)$ de la commune de Pagolle (1297). Corpus : 151 cartes lexicales. Indice de distance $R I W_{j k}$. Algorithme de regroupement MINMWMAX 12 groupes. 


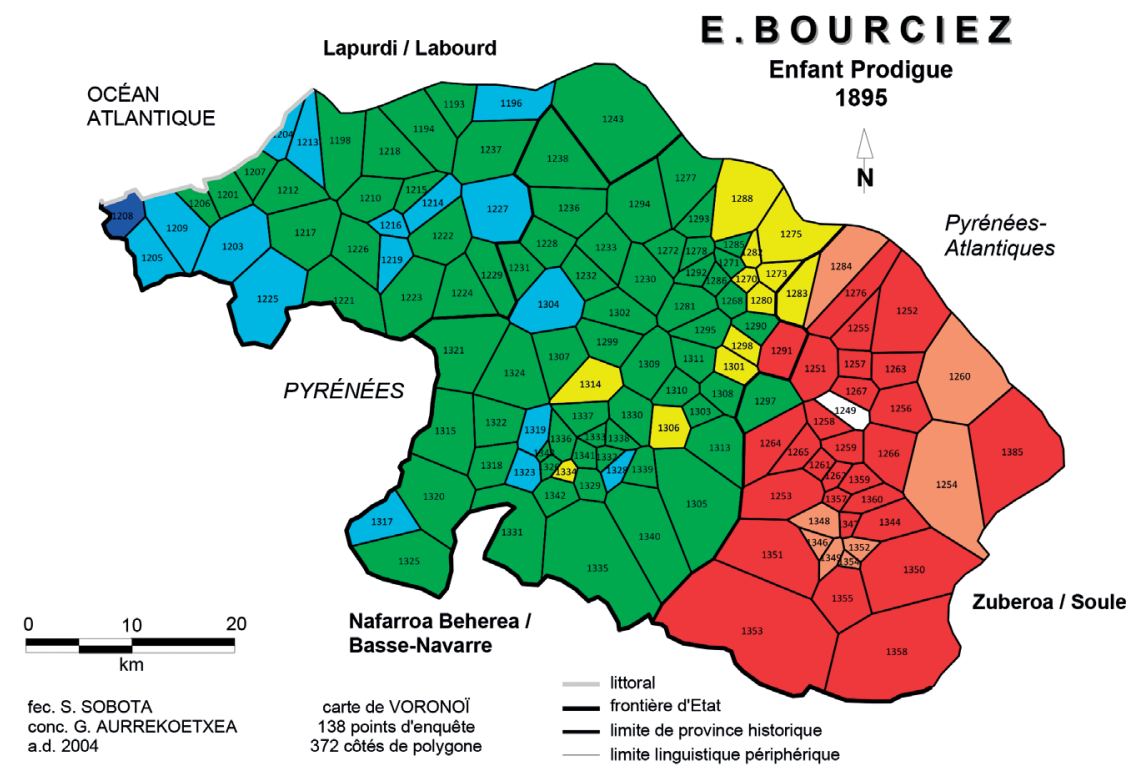

Algorithme de regroupement MINMWMAX 6 groupes

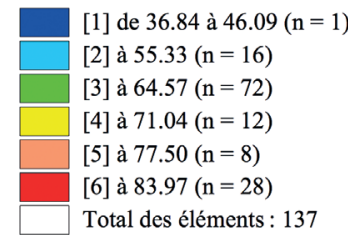

Carte 8. - Carte choroplèthe des distributions d'indice de similarité $\left(R I W_{1297, k}\right)$ de la commune de Mauléon (1249). Corpus : 151 cartes lexicales. Indice de distance $R I W_{j k}$. Algorithme de regroupement MINMWMAX 12 groupes. 


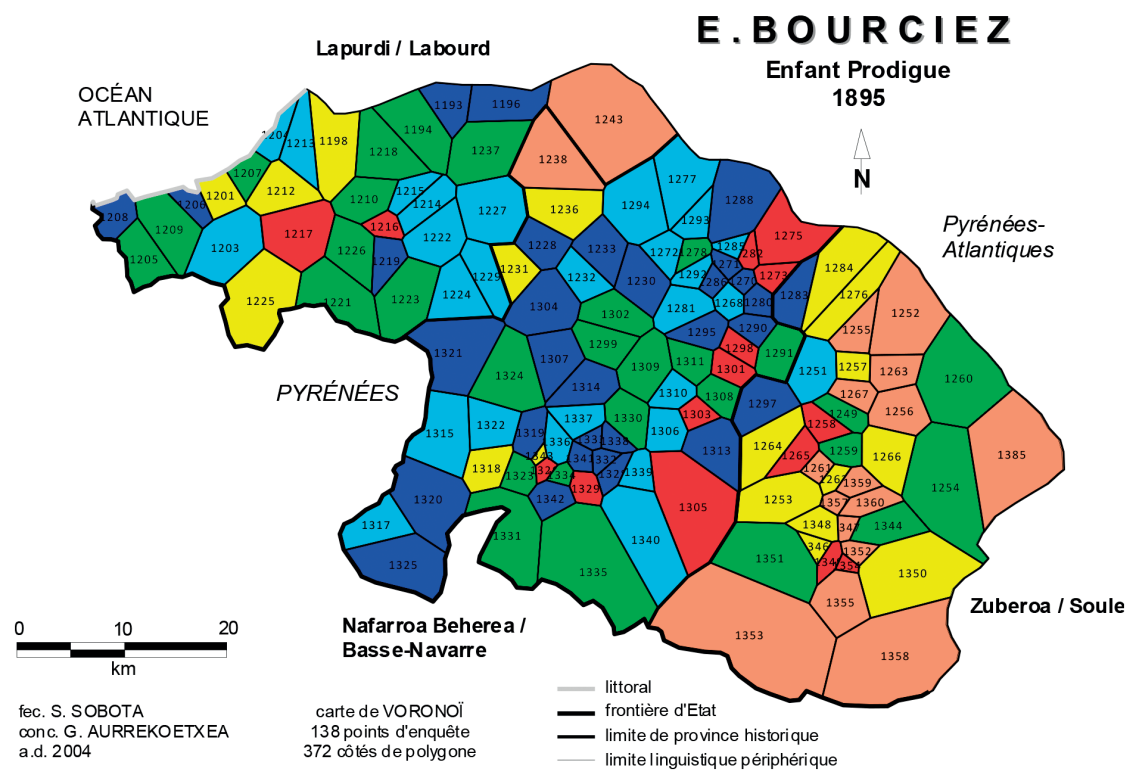

\section{Algorithme de regroupement MedMw 6 groupes}

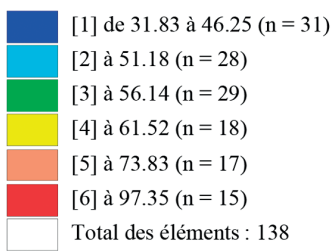

Distribution de la fréquence de similarité MedMw 12 groupes

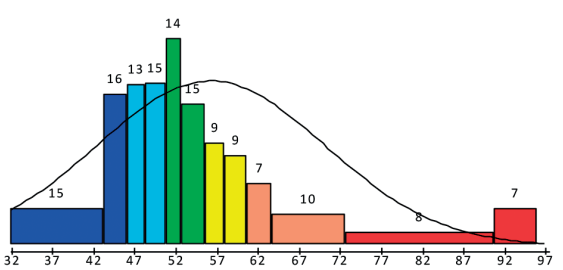

Carte 9. - Carte choroplèthe des synopsies des distributions maximales. Corpus : lexique total des 151 cartes; carte obtenue à partir de l'indice de similarité $G I W(1)_{j k}$ et de l'algorithme de regroupement MEDMW 6 groupes (6 couleurs sur la carte). 


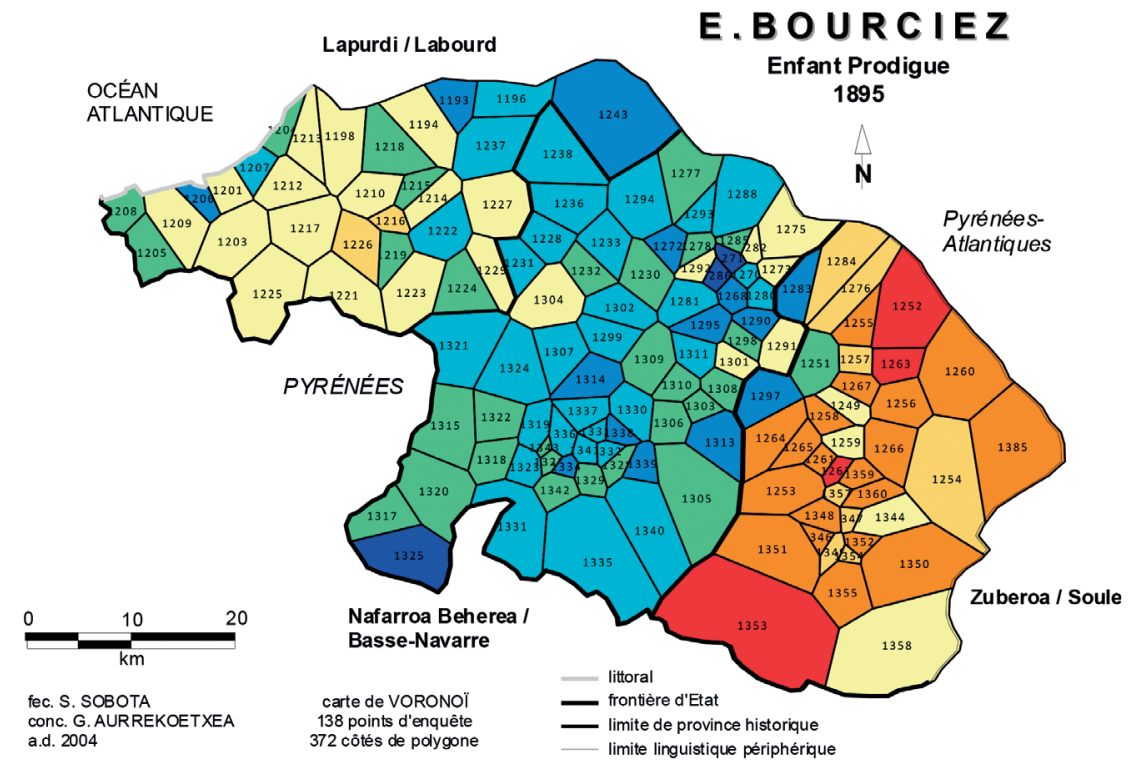

Algorithme de regroupement MINMWMAX 8 groupes

Distribution de la fréquence de similarité MINMWMAX 16 groupes

[1] de 2.71 à $3.36(n=3)$

[2] à $4.01(n=14)$

[3] à $4.65(n=32)$

[4] à $5.30(n=30)$

[5] à $6.05(n=26)$

[6] à $6.80(\mathrm{n}=10)$

[7] à $7.55(n=19)$

[8] à $8.30(n=4)$

Total des éléments : 138

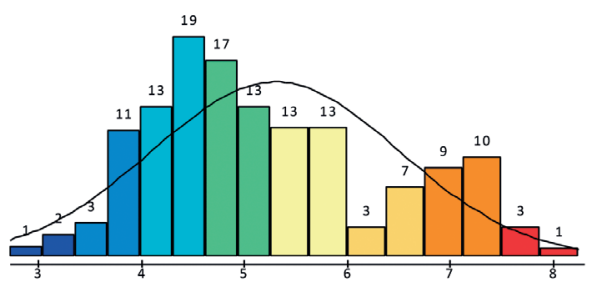

Carte 10. - Carte des écarts-types : desbideratze tipikoaren mapa. Corpus : lexique des 151 cartes. Indice de similarité $R I W_{j k}$. Algorithme de regroupement MINMWMAX 8 groupes. 


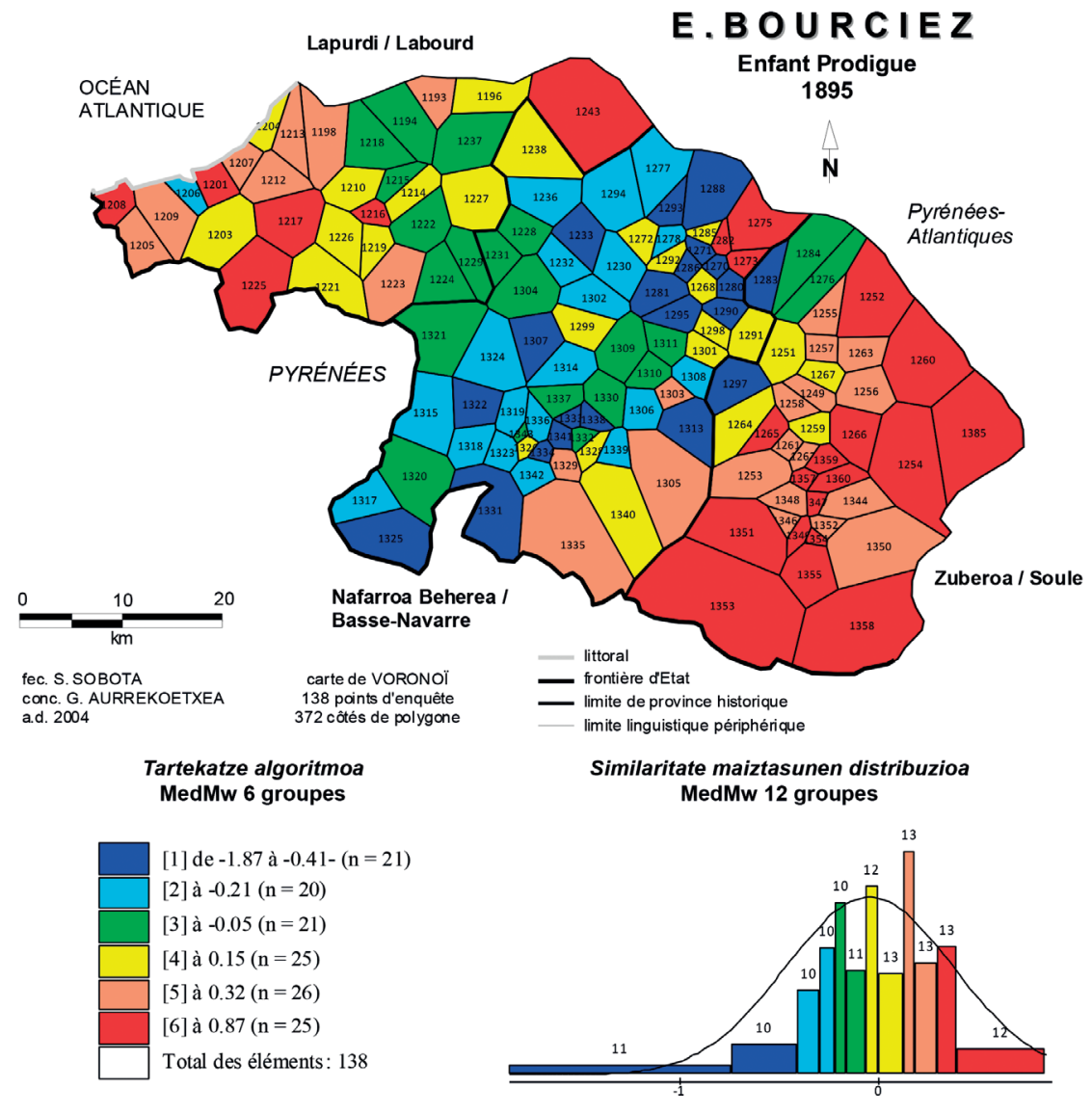

Carte 11. - Carte choroplèthe de synopsie du coefficient d'asymétrie de Fischer. Corpus : lexique des 151 cartes. Indice de similarité IRI ${ }_{j k}$. Algorithme de regroupement MEDMW 6 groupes. 


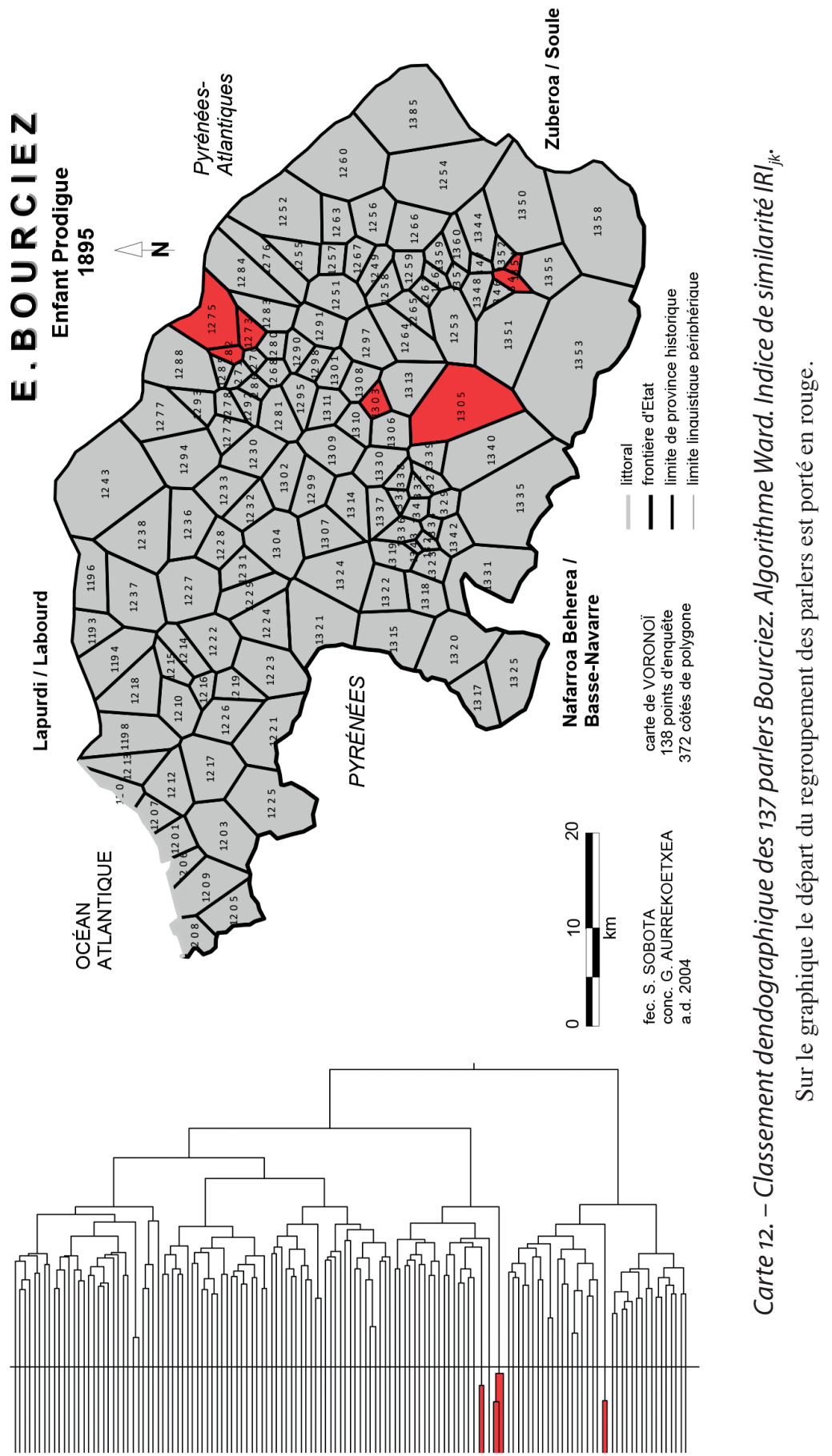




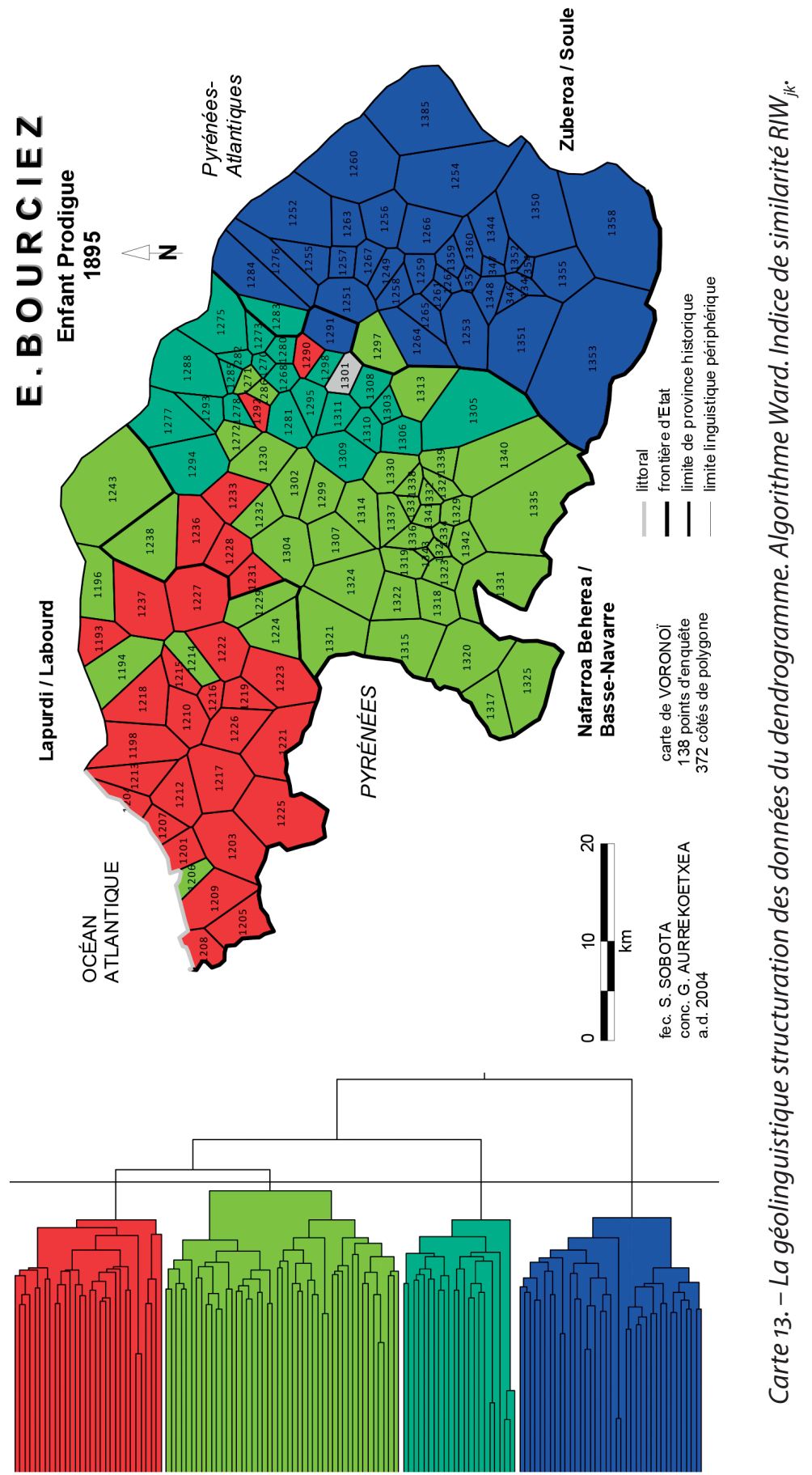




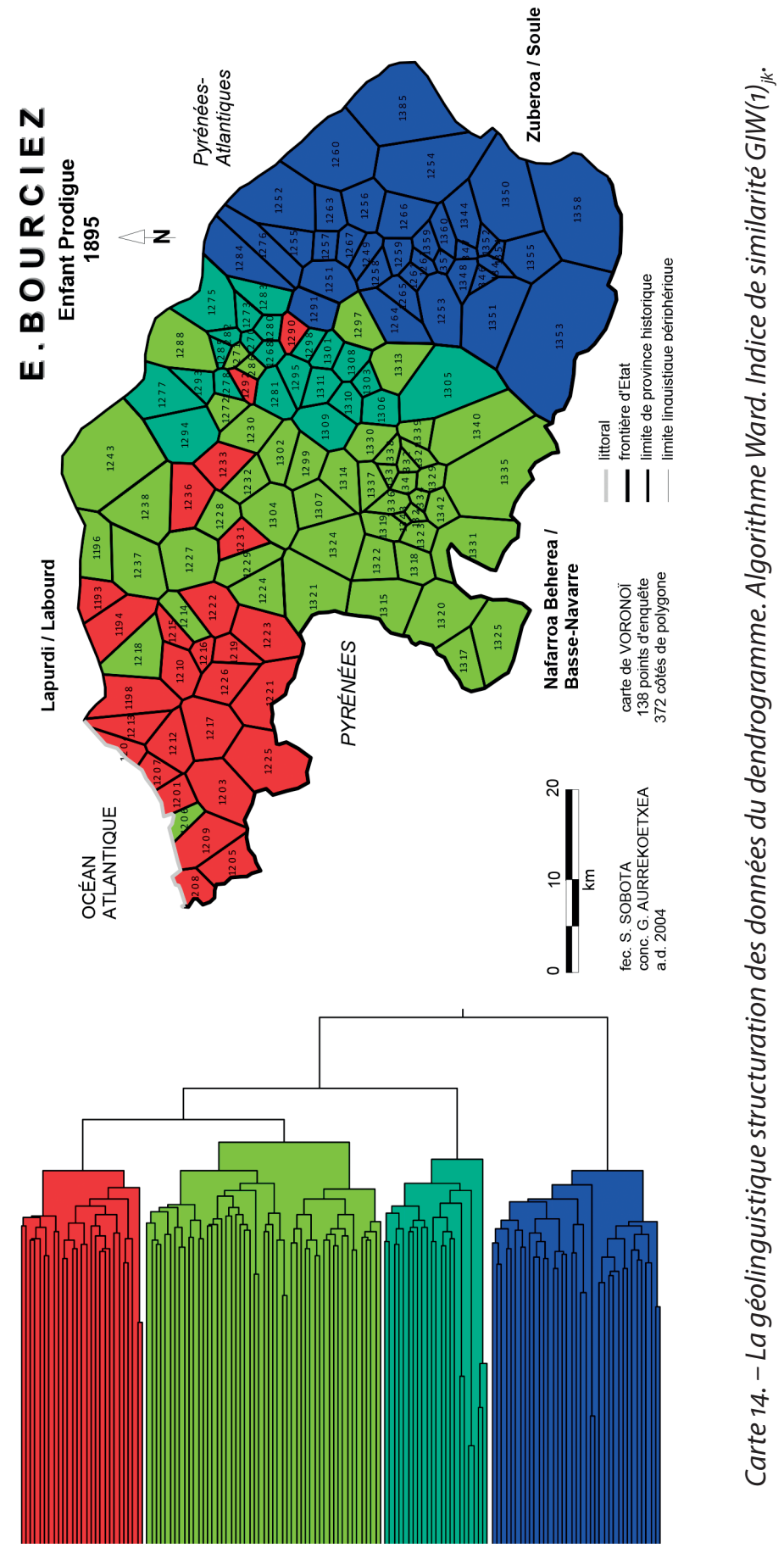




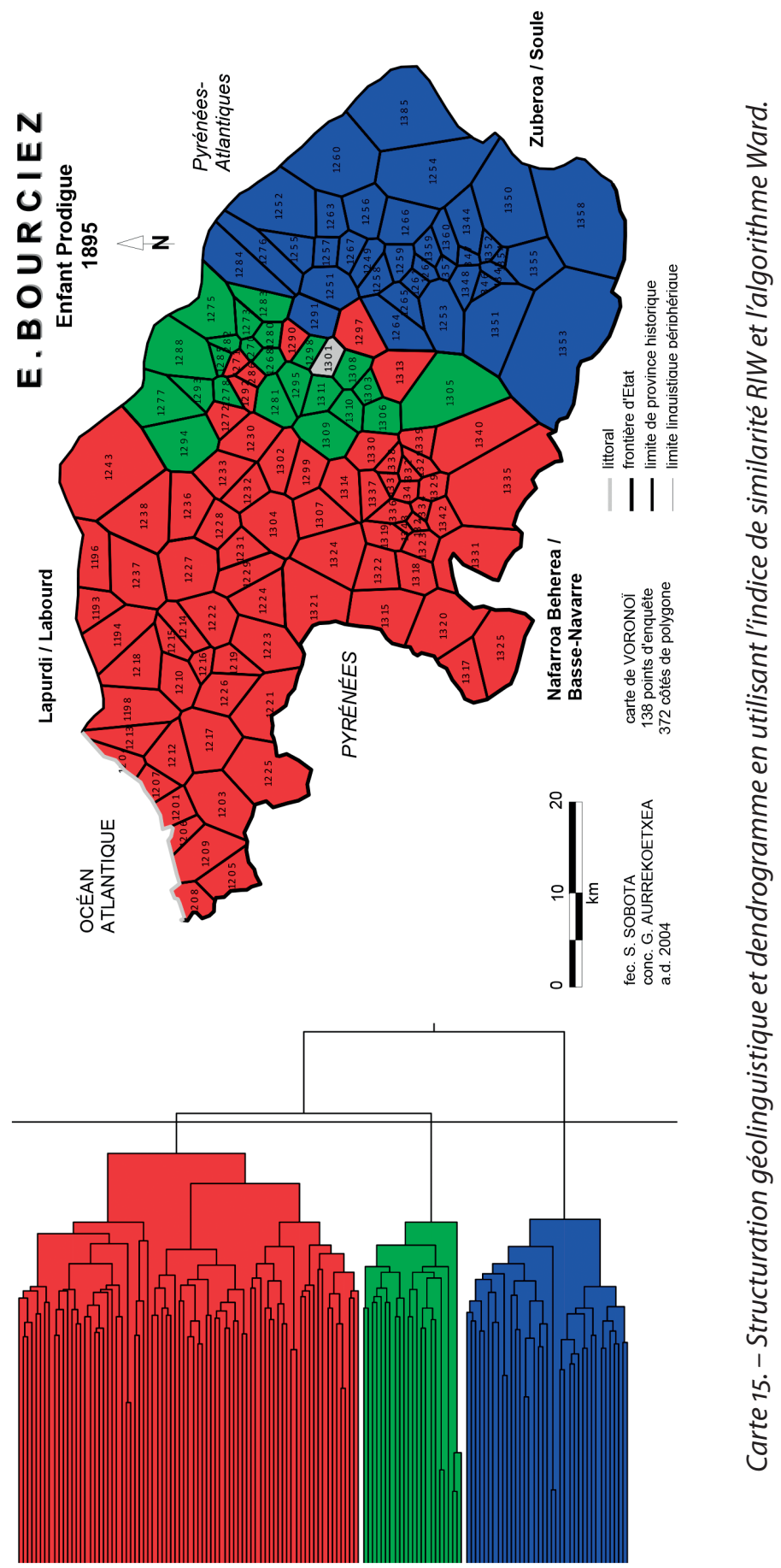

